\title{
Some aspects of the $\boldsymbol{m}$-adic analysis and its applications to $m$-adic stochastic processes
}

\author{
M.V. Dolgopolov, * \\ and
}

A.P. Zubarev ${ }^{\dagger}$

November 8, 2018

\begin{abstract}
In this paper we consider a generalization of analysis on $p$-adic numbers field to the $\mathrm{m}$ case of $m$-adic numbers ring. The basic statements, theorems and formulas of $p$-adic analysis can be used for the case of $m$-adic analysis without changing. We discuss basic properties of $m$-adic numbers and consider some properties of $m$-adic integration and $m$-adic Fourier analysis. The class of infinitely divisible $m$-adic distributions and the class of $m$-adic stochastic Levi processes were introduced. The special class of $m$-adic CTRW process and fractional-time $m$-adic random walk as the diffusive limit of it is considered. We found the asymptotic behavior of the probability measure of initial distribution support for fractional-time $m$-adic random walk.
\end{abstract}

\section{Introduction}

Up to recent time, ultrametric spaces were known only in mathematical literature. A classic example of such space is the $p$-adic numbers [1, 2] field. During the last three decades the interest in ultrametric models research has risen in various fields of physics, biology, economics and sociology, such as models of spin glass, biopolymers, fractal structures as well as in optimization theory, taxonomy, evolutional biology, cluster and factor analysis etc. (refer to the surveys [3] and [4). In recent years the scientific interest laid also in the field of the dynamic models for the ultrametric structures, in particular the models of ultrametric diffusion (see [5, 6, 7, 8, 9] and references therein), which are directly related to the description of the conformational rearrangements of the proteins dynamics as well, apparently may be related

\footnotetext{
*Samara State University, e-mail: mikhaildolgopolov@rambler.ru
}

${ }^{\dagger}$ Samara State University, e-mail: apzubarev@mail.ru 
to the random processes modeling in the complicated biological and socio-economic systems [10, 11, 12].

The ultrametricity of space is closely related to a concept of hierarchic structure over space [3]. For complex systems modeling hierarchic structures may appear both on the space of states of the system and on the space of objects constituting the system. E. g., one describes dynamics of conformation rearrangements of biopolymer macromolecules, multiple local minima of free energy can be regarded as highly degenerated space of the system states possessing the hierarchic structure [5, 6, 7, 8].

An adequate mathematical tools to formalize description of mathematical modeling of the complex systems hierarchic structures are required. One such tool is a $p$-adic analysis developed during the last three decades by the group of Academician V.S. Vladimirov in Steklov Mathematical Institute. The main idea of application of $p$-adic analysis at mathematical modeling of complex hierarchically organized systems is that the field of $p$-adic numbers $\mathbf{Q}_{p}$ has a natural regular indexed hierarchic structure with the degree of regularity equal to certain prime number $p$ and thus a configuration space of such systems can be adequately described by $p$-adic coordinate space.

Nevertheless, it is worth to mention that, strictly speaking, the mathematical tools of $p$-adic analysis is directly applicable for describing a hierarchically organized system only in the case when the system possesses regular indexed hierarchic structure and degree of regularity of the hierarchic structure is a prime number $p$. The analysis on general ultrametric spaces described by oriented trees has been developed in [13]. Nevertheless, for specific computations it is necessary to use explicit parametrization of the points of an ultrametric space, in which the computational results are represented. For example, in the case of $\mathbf{Q}_{p}$ such parametrization has the form of $a_{n} p^{n}+a_{n-1} p^{n-1}+\ldots$. The using of such a parametrization for ultrametric space corresponding to an oriented tree of general type is rather difficult.

In this paper, which has partly methodological in nature, we consider a generalization an analysis on a field $\mathbf{Q}_{p}$ for more general case of ring of $m$-adic numbers $\mathbf{Q}_{m}$. The ring $\mathbf{Q}_{m}$ as well as the field $\mathbf{Q}_{p}$ is local-compact Abelian group. Thus for $\mathbf{Q}_{m}$ there exists a unique (up to a normalization) translation-invariant Haar measure and dual group of characters which allows to develop Fourier analysis and consider the classes of pseudo-differential operators. We show that the basic statements, theorems and formulas of $p$-adic analysis [2, 14] can be used for the case of $m$-adic analysis without changing. In section 2 we discuss basic properties of $m$-adic numbers $\mathbf{Q}_{m}$. In sections 3 and 4 we consider some properties of $m$-adic integration and $m$-adic Fourier analysis. In section 5 we introduce a class of infinitely divisible $m$-adic distributions and a class of $m$-adic random Levi processes. In section 6 we consider the special class of random processes of uncoupled continuous time random walk type on $\mathbf{Q}_{m}(m$-adic CTRW). In section 7 we discuss the diffusive limit of $m$-adic CTRW together with the equation of fraction-time $m$-adic random walk. In section 8 we find the asymptotic behavior of probability measure of initial distribution support for a random process described by fraction-time equation of $m$-adic random walk. 


\section{The ring of $m$-adic numbers $\mathrm{Q}_{m}$}

Let us define a ring $\mathbf{Q}_{m}$ as the set of infinite series

$$
x=\sum_{i=r}^{\infty} a_{i} m^{i},
$$

where $r$ is an integer number, $a_{r}=1,2,3, \ldots, m-1, a_{i}=0,1,2,3, \ldots, m-1(i \neq r)$. The set $\mathbf{Q}_{m}$ has a structure of commutative associative ring, i.e. the sum and product operations are defined in it. Let us call a sum of two $m$-adic numbers $x=\sum_{i=r}^{\infty} a_{i} m^{i}$ and $y=\sum_{i=s}^{\infty} b_{i} m^{i}$ such element $z=\sum_{i=l}^{\infty} c_{i} m^{i}, l=\min \{r, s\}$ that

$$
\sum_{i=r}^{\infty} a_{i} m^{i}+\sum_{i=s}^{\infty} b_{i} m^{i}=\sum_{i=l}^{\infty} c_{i} m^{i} .
$$

Let us call the product of two $m$-adic numbers $x=\sum_{i=r}^{\infty} a_{i} m^{i}$ and $y=\sum_{i=s}^{\infty} b_{i} m^{i}$ such an element $z=\sum_{i=l}^{\infty} c_{i} m^{i}$ that $l \geq r+s$ and

$$
\sum_{i=r}^{\infty} \sum_{j=s}^{\infty} a_{i} b_{j} m^{i} m^{j}=\sum_{i=r+s}^{\infty} c_{i} m^{i} .
$$

Comparing right and left parts of equations (2.1) and (2.2) by $\bmod m^{n}$ for $n=$ $1,2,3, \ldots$ makes it possible to find successively the coefficients $c_{i}$ for the sum $z=x+y$ and product $z=x y$.

Division operation in $\mathbf{Q}_{m}$ is uniquely defined only in case $m$ is a prime $p$. In such case, the ring $\mathbf{Q}_{m}$ is a field of $p$-adic numbers $\mathbf{Q}_{p}$.

There exists $m$-adic pseudonorm $|x|_{m}=m^{-r}$ in $\mathbf{Q}_{m}$ for $x=\sum_{i=r}^{\infty} a_{i} m^{i}$ which satisfies the following properties:

1. $|x|_{m}=0 \Leftrightarrow x=0$,

2. $|x y|_{m} \leq|x|_{m}|y|_{m}$,

3. $|x+y|_{m} \leq \max \left\{|x|_{m},|y|_{m}\right\}$.

In the case $m$ is a prime $p$, non-eq. 2. transforms into eq. $|x y|_{p}=|x|_{p}|y|_{p}$, and $m$ adic pseudonorm $|x|_{m}$ transforms into $p$-adic norm $|x|_{p}$. Note that for any $x \in \mathbf{Q}_{m}$ and any $r \in \mathbf{Z}$, the following equation takes place:

$$
\left|m^{r} x\right|_{m}=m^{-r}|x|_{m}
$$

Pseudonorm $|x|_{m}$ induces metrics $d_{m}(x, y)=|x-y|_{m}$ satisfying the following properties:

1. $d_{m}(x, y)=0$ then and only then if $x=y$,

2. $d_{p}(x, y) \leq \max \left\{d_{p}(x, z), d_{p}(y, z)\right\}$ for any $x, y$ and $z$ from $\mathbf{Q}_{m}$. 
We call the subset $B_{r}\left(x_{0}\right)=\left\{x \in \mathbf{Q}_{m}:\left|x-x_{0}\right| \leq m^{r}\right\}$ a disk of radius $m^{r}$ with the center at the point $x_{0} \in \mathbf{Q}_{m}$, and subset $S_{r}\left(x_{0}\right)=\left\{x \in \mathbf{Q}_{m}:\left|x-x_{0}\right|=m^{r}\right\}-$ a circumference of radius $m^{r}$ with the center at the point $x_{0} \in \mathbf{Q}_{m}$. Also we denote $B_{r}(0) \equiv B_{r}, S_{r}(0) \equiv S_{r}, B_{0} \equiv Z_{m}$. Due to the same metric structure of both $\mathbf{Q}_{m}$ and $\mathbf{Q}_{p}$ the following statements for $\mathbf{Q}_{m}$ take place [2]:

1. All disks and circumferences in $\mathbf{Q}_{m}$ are closed and open sets.

2. All points of a disk is also its center.

3. If two disks have a common point, then one of them is contained in another.

4. All sets open in $\mathbf{Q}_{m}$ are countable unions of nonintersecting disks.

5. $\mathrm{Q}_{m}$ has zero dimension.

6. $\mathbf{Q}_{m}$ is separable totally disconnected, Hausdorf, locally compact.

\section{Measure and integration on $\mathrm{Q}_{m}$}

As $\mathbf{Q}_{m}$ is a local compact commutative group there exist an additive translation invariant measure $\mu\left(B \in \mathbf{Q}_{m}\right)$ [2]. This measure is uniquely determined by properties:

1. $\mu\left(Z_{m}\right)=1$ (normalization),

2. $\forall a \in \mathrm{Q}_{m}, \forall B \in \mathrm{B} \mu(B)=\mu(B+a)$ (translational invariance),

3. $\forall\left\{B_{i} \in \mathrm{B}\right\}, B_{i} \bigcap B_{j}=\emptyset, i \neq j \Rightarrow \mu\left(\bigcup_{i} B_{i}\right)=\sum_{i} \mu\left(B_{i}\right)$ (additivity).

Note that the measure satisfies the following property $\mu\left(m^{r} A\right)=m^{-r} \mu(A)$ but in general $\mu(x A) \neq|x|_{m} \mu(A)$. For example in the case $m=4$ we have $2 \cdot B_{0} \subset B_{0}$. On the other hand

$$
\begin{gathered}
2 \cdot B_{0}=2 \cdot\left(\left(0+B_{-1}\right) \bigcup\left(1+B_{-1}\right) \bigcup\left(2+B_{-1}\right) \bigcup\left(3+B_{-1}\right)\right)= \\
=\left(2 \cdot B_{-1}\right) \bigcup\left(2+2 \cdot B_{-1}\right) \bigcup\left(4+2 \cdot B_{-2}\right) \bigcup\left(2+4+B_{-1}\right),
\end{gathered}
$$

therefore $3+B_{-1} \not \subset 2 \cdot B_{0}$ and $2 \cdot B_{0} \subset B_{0} \backslash\left(3+B_{-1}\right)$. By additivity $\mu\left(2 \cdot B_{0}\right) \leq$ $\mu\left(B_{0}-\left(3+B_{-1}\right)\right)=\mu\left(B_{0}\right) \backslash \mu\left(3+B_{r-1}\right)=4^{r}-4^{r-1}$ and $\mu\left(2 \cdot B_{r}\right) \neq|2|_{4} \mu\left(B_{r}\right)=$ $4^{r}$.

For $B \subset \mathbf{Q}_{m}$ let us call

$$
\mu^{\star}(B)=\inf _{B \subset \cup B_{i}} \sum_{i} \mu_{i}\left(B_{i}\right)
$$

an external measure, where the infimum is taken over all possible coverings of the $B$ by countable set of non-intersecting disks $\left\{B_{i}\right\}$. $B$ is measurable if for any $\varepsilon>0$ it is possible to find such elementary multiplicity $A$ that $\mu^{\star}((B \bigcup A) \backslash(B \cap A))<\varepsilon$. Let $\mathrm{B}$ be the class of all measurable subsets of $\mathbf{Q}_{m}$. Then for any $B \in \mathrm{B} \mu(B) \equiv \mu^{*}(B)$. 
It is possible to prove (e.g. refer to [16]) that B is $\sigma$-algebra and the measure $\mu(B)$ is $\sigma$-additive on $\mathrm{B}$.

A complex valued function $f(x)$ on $B \in \mathrm{B}$ is measurable if for any Borelean $f^{-1}(\mathrm{~B})$ it is measurable set. For measurable functions $f(x)$ it is possible to define linear continuous functional - integral $\int_{B} f(x) d_{m} x$ of the function $f(x)$ over the measure $\mu(B)$.

The set of measurable on $B$ functions for which the integral $\int_{B}|f(x)|^{\lambda} d_{m} x$ at $\lambda \geq 1$ is finite, will be denoted by $L^{\lambda}(B)$. Let $f(x) \in L_{\text {loc }}^{\lambda}\left(\mathbf{Q}_{m}\right)$ if for any measurable compact set $B \in \mathbf{Q}_{m} f(x) \in L^{\lambda}(B)$.

A convolution $(f * g)(x)$ of two functions is

$$
(f * g)(x)=\int_{\mathbf{Q}_{m}} f(y) g(x-y) d_{m} y .
$$

Theorem 3.1. Let the functions $f(x)$ and $g(x)$ are continuous and limited on $\mathbf{Q}_{m}, f(x) \in L^{1}\left(\mathbf{Q}_{m}\right), g(x) \in L^{1}\left(\mathbf{Q}_{m}\right)$. Then $(f * g)(x)$ exists for any $x$. Moreower, $(f * g)(x)$ is continuous and limited, $(f * g)(x) \in L^{1}\left(\mathbf{Q}_{m}\right)$ and

$$
\int_{\mathbf{Q}_{m}}(f * g)(x) d_{m} x=\int_{\mathbf{Q}_{m}} f(x) d_{m} x \int_{\mathbf{Q}_{m}} g(x) d_{m} x .
$$

The proof is the same as in regular analysis (e.g. see [17]).

Below some integrals, that we need later, are present.

If a function $f(x)$ depends only on $|x-a|_{m}$, then the integral of it over disk $B_{r}(a)$ equals to

$$
\int_{B_{r}(a)} f\left(|x-a|_{m}\right) d_{m} x=\left(1-m^{-1}\right) \sum_{i=-\infty}^{r} m^{i} f\left(m^{i}\right) .
$$

Let us define the function

$$
\chi_{m}(x)=\exp (2 \pi i\{x\})
$$

where $\{x\}$ is fraction part of $x$ defined as

$$
\left\{\sum_{i=r}^{\infty} a_{i} m^{i}\right\}=\left\{\begin{array}{l}
\sum_{i=r}^{-1} a_{i} m^{i}, r<0 \\
0, r \geq 0
\end{array}\right.
$$

Let us evaluate the integral $\int_{B_{r}} \chi_{m}(k x) d_{m} x$ where $k \in \mathbf{Q}_{m}$. At $|k|_{m} \leq m^{-r}|k x|_{m} \leq$ $|k|_{m}|x|_{m} \leq 1$ and $\chi_{m}(x)=1$, therefore $\int_{B_{r}} \chi_{m}(k x) d_{m} x=m^{r}$. At $|k|_{m}>m^{-r}$ we shall make a substitution $x \rightarrow y=x-m^{r}, B_{r} \rightarrow B_{r}\left(p^{r}\right)=B_{r}$. Then

$$
\int_{B_{r}} \chi_{m}(k x) d_{m} x=\int_{B_{r}} \chi_{m}\left(k y+k m^{r}\right) d_{m} y=\chi_{m}\left(k m^{r}\right) \int_{B_{r}} \chi_{m}(k y) d_{m} y
$$


and we obtain

$$
\left(1-\chi_{m}\left(k m^{r}\right)\right) \int_{B_{r}} \chi_{m}(k y) d_{m} y=0 .
$$

As $|k|_{m}>m^{-r}($ see (2.3) $)$ we have $\left|k m^{r}\right|_{m}=|k|_{m}\left|m^{r}\right|_{m}=|k|_{m} m^{-r}>1$. By (3.2)-(3.3) $\left(1-\chi_{m}\left(k^{r}\right)\right) \neq 0$ hence from (3.4) it follows that $\int_{B_{r}} \chi_{m}(k y) d_{m} y=0$. Therefore we have

$$
\int_{B_{r}} \chi_{m}(k y) d_{m} y=\left\{\begin{array}{l}
m^{r},|k|_{m} \leq m^{-r} \\
0,|k|_{m}>m^{-r} .
\end{array}\right.
$$

From (3.5) it follows that

$$
\int_{S_{r}} \chi_{m}(k y) d_{m} y=\left\{\begin{array}{l}
m^{r}\left(1-m^{-1}\right),|k|_{m} \leq m^{-r} \\
-m^{r-1},|k|_{m}=m^{-r+1}, \\
0,\left.k\right|_{m}>m^{-r+1} .
\end{array}\right.
$$

It is not difficult to find for any $f\left(|x|_{m}\right) \in L_{\mathrm{loc}}^{1}\left(\mathbf{Q}_{m}\right)$

$$
\begin{gathered}
\int_{B_{r}} f\left(|x|_{m}\right) \chi_{m}(k x) d_{m} x= \\
=\left\{\begin{array}{c}
\left(1-m^{-1}\right) \sum_{r=-s}^{\infty} m^{-r} f\left(m^{-r}\right),|k|_{m} \leq m^{-s}, \\
\left(1-m^{-1}\right) \frac{1}{|k|_{m}} \sum_{r=0}^{\infty} m^{-r} f\left(\frac{m^{-r}}{|k|_{m}}\right)-\frac{1}{|k|_{m}} f\left(\frac{m}{|k|_{m}}\right),|k|_{m}>m^{-s} .
\end{array}\right.
\end{gathered}
$$

Note that the integrals (3.1), (3.5), (3.6) and (3.7) coincide with corresponding integrals on $\mathbf{Q}_{p}[2$ after substitution $p \rightarrow m$.

\section{Fourier analysis on $\mathrm{Q}_{m}$}

The character of $\mathbf{Q}_{m}$ as an abelian additive group is a complex-value function $\chi(x)$ on $\mathbf{Q}_{m}$ with properties:

1. $|\chi(x)|=1$,

2. $\chi(x)=1 \Leftrightarrow x=0$,

3. $\forall x, y \in Q_{m} \chi(x+y)=\chi(x) \chi(y)$.

Theorem 4.1. The group $\mathbf{Q}_{m}$ of characters on group $\mathbf{Q}_{m}$ is isomorphous to $\mathbf{Q}_{m}$, and arbitrary element $\chi(x)$ from $\mathbf{Q}_{m}$ is given by $\chi_{m}(k x)=\exp (2 \pi i\{k x\})$, $k \in \mathbf{Q}_{m}$. 
Proof. Consider some character $\chi(x)$. Let $\int_{B_{r}} \chi(x) d_{m} x=c$. If $c \neq 0$, then for all $y \in B_{r}$ from the equation

$$
\int_{B_{r}} \chi\left(x^{\prime}\right) d_{m} x^{\prime}=\int_{B_{r}} \chi(x+y) d_{m}(x+y)=\chi(y) \int_{B_{r}} \chi(x) d_{m} x
$$

it follows that $\chi(y)=1$. Let $s=\max \left\{r: \int_{B_{r}} \chi(x) d_{m} x \neq 0\right\}$. Note that $s$ exists, if $\chi(x) \neq 1 \forall x$. Consider arbitrary $x \in \mathbf{Q}_{m}$

$$
x=m^{-r}\left(b_{0}+m b_{1}+m^{2} b_{2}+\ldots+m^{r-s-1} b_{r-s-1}\right)+m^{-s}\left(a_{0}+m a_{1}+m^{2} a_{2}+\ldots\right)
$$

As $\chi\left(B_{s}\right)=1$ we get

$$
\chi(x)=\left(\chi\left(m^{-r}\right)\right)^{b_{0}}\left(\chi\left(m^{-r+1}\right)\right)^{b_{1}} \ldots\left(\chi\left(m^{-s-1}\right)\right)^{b_{r-s-1}}
$$

After some transformations

$$
\begin{gathered}
\chi\left(m^{-r}\right)=\chi\left(m^{-s+s-r}\right)=\left(\chi\left(m^{-s}\right)\right)^{m^{s-r}}=\exp \left(2 \pi i\left(m^{-r+s} l\right)\right), \\
\chi\left(m^{-r+1}\right)=\chi\left(m^{-r}\right)^{m}=\exp \left(2 \pi i\left(m^{-r+s+1} l\right)\right), \\
\cdots \\
\chi\left(m^{-s-1}\right)=\left(\chi\left(m^{-r}\right)\right)^{m^{r-s-1}}=\exp \left(2 \pi i\left(m^{-1} l\right)\right),
\end{gathered}
$$

where $l=0,1, \ldots, m^{r-s}-1$, we get

$$
\chi(x)=\exp \left(2 \pi i\left(\left(l m^{s}\right)\left(m^{-r} b_{0}+m^{-r+1} b_{1}+\ldots+m^{-s-1} b_{r-s-1}\right)\right)\right) .
$$

By denoting $l m^{s} \equiv k$ and expanding $k=m^{s}\left(k_{0}+m k_{1}+\ldots+m^{r-s-1} k_{r-s-1}\right)$ we have

$$
\begin{gathered}
\chi(x)= \\
=\exp \left[2 \pi i \left(\left(m^{s}\left(k_{0}+m k_{1}+\ldots+m^{r-s-1} k_{r-s-1}\right)\right) \times\right.\right. \\
\left.\left.\left(m^{-r}\left(b_{0}+m b_{1}+\ldots+m^{r-s-1} b_{r-s-1}\right)\right)\right)\right] .
\end{gathered}
$$

Thus every character for $\forall x \in \mathbf{Q}_{m}$ is defined by $k \in \mathbf{Q}_{m}$ and has the form

$$
\chi(x)=\exp (2 \pi i k x)=\exp (2 \pi i\{k x\}) .
$$

Let $f(x) \in L^{1}\left(\mathbf{Q}_{m}\right)$, then for any $k \in \mathbf{Q}_{m} f(x) \chi_{m}(k x) \in L^{1}\left(\mathbf{Q}_{m}\right)$. Let us call $m$-adic Fourier transform of a function $f(x)$ such function $\tilde{f}(k)$ that

$$
\tilde{f}(k)=\int_{\mathbf{Q}_{m}} \chi_{m}(k x) f(x) d_{m} x .
$$

Function $f(x)$ on $\mathbf{Q}_{m}$ is called locally constant if $f(x)$ is finite for all $x \in \mathbf{Q}_{m}$, and for all $x \in \mathbf{Q}_{m}$ there exists integer $l(x)$ that $f\left(x+x^{\prime}\right)=f(x)$ for any $x^{\prime} \in B_{l}(x)$. If there exists integer $l$ such that for all $x \in \mathbf{Q}_{m}$ and all $x^{\prime} \in B_{l}$ equation $f\left(x+x^{\prime}\right)=f(x)$, 
then $f(x)$ is called locally constant on $\mathbf{Q}_{m}$ with constancy parameter $l$. Let us implement the following designations:

$E\left(\mathbf{Q}_{m}\right)$ - set of functions locally constant on $\mathbf{Q}_{m}$;

$E^{l}\left(\mathbf{Q}_{m}\right)$ - set of functions locally constant on $\mathbf{Q}_{m}$ with constancy parameter $l$;

$D\left(\mathbf{Q}_{m}\right)$ - set of functions on $\mathbf{Q}_{m}$ with compact support;

$D_{r}\left(\mathbf{Q}_{m}\right)$ - set of functions on $\mathbf{Q}_{m}$ with a support in a disk $B_{r}$;

$D_{r}^{l}\left(\mathbf{Q}_{m}\right)$ - set of functions locally constant on $\mathbf{Q}_{m}$ with support in disk $B_{r}$ and constancy parameter $l$.

Theorem 4.2. If $f(x) \in D_{r}^{l}\left(\mathbf{Q}_{m}\right)$, then $\tilde{f}(k) \in D_{-l}^{-r}\left(\mathbf{Q}_{m}\right)$.

Proof. Let $k^{\prime} \in B_{-r}$. Then

$$
\tilde{f}\left(k+k^{\prime}\right)=\int_{\mathbf{Q}_{m}} \chi_{m}\left(k x+k^{\prime} x\right) f(x) d_{m} x=\int_{B_{r}} \chi_{m}(k x) \chi_{m}\left(k^{\prime} x\right) f(x) d_{m} x
$$

Because in the integrand $\left|k^{\prime} x\right|_{m} \leq\left|k^{\prime}\right|_{m}|x|_{m} \leq 1$, then $\chi_{m}\left(k^{\prime} x\right)=1$, we have $\tilde{f}(k+$ $\left.k^{\prime}\right)=\int_{B_{r}} \chi_{m}(k x) f(x) d_{m} x=\int_{\mathbf{Q}_{m}} \chi_{m}(k x) f(x) d_{m} x=\tilde{f}\left(k^{\prime}\right)$ and $\tilde{f}(k) \in \varepsilon^{-r}\left(\mathbf{Q}_{m}\right)$. Let $|k|_{m}>m^{-l}$. Then after substitution $x \rightarrow y=x-m^{-l}$ we get

$$
\begin{gathered}
\tilde{f}(k)=\int_{\mathbf{Q}_{m}} \chi_{m}(k x) f(x) d_{m} x=\int_{\mathbf{Q}_{m}} \chi_{m}\left(k y+k m^{-l}\right) f\left(y+m^{-l}\right) d_{m} y= \\
=\chi_{m}\left(k m^{-l}\right) \int_{\mathbf{Q}_{m}} \chi_{m}(k y) f(y) d_{m} y=\chi_{m}\left(k m^{-l}\right) \tilde{f}(k) .
\end{gathered}
$$

Because $\left|k m^{-l}\right|_{m}=|k|_{m}\left|m^{-l}\right|_{m}=|k|_{m} m^{l}>1$, we have $\left(1-\chi_{m}\left(k m^{-l}\right)\right) \neq 0$ hence $\tilde{f}(k)=0$ and $\tilde{f}(k) \in D_{-l}^{-r}\left(\mathbf{Q}_{m}\right)$.

Theorem 4.3. Let the function $f(x)$ is continuous on $\mathbf{Q}_{m}$ and $f(x) \in L^{1}\left(\mathbf{Q}_{m}\right)$. Then the inversion formula is hold:

$$
f(x)=\int_{\mathbf{Q}_{m}} \chi_{m}(-k x) \tilde{f}(k) d_{m} k .
$$

Proof. Consider

$$
f_{r}(x) \equiv \int_{B_{r}} \chi_{m}(-k x)\left(\int_{\mathbf{Q}_{m}} \chi_{m}(k y) f(y) d_{m} y\right) d_{m} k
$$

Let us show that $\lim _{r \rightarrow \infty} f_{r}(x)=f(x)$. By uniform convergence of the internal integral we have

$$
\begin{aligned}
f_{r}(x) & =\int_{\mathbf{Q}_{m}} \int_{B_{r}} \chi_{m}(k(y-x)) d_{m} k f(y) d_{m} y= \\
& =m^{r} \int_{\mathbf{Q}_{m}} \Omega\left(m^{r}|y-x|_{m}\right) f(y) d_{m} y,
\end{aligned}
$$


where $\Omega(\lambda)=\left\{\begin{array}{l}1, \lambda \leq 1, \\ 0, \lambda>1 .\end{array}\right.$ Taking into account $m^{-r} \int_{\mathbf{Q}_{m}} \Omega\left(m^{r}|y-x|_{m}\right) d_{m} y=1$, we have

$$
\begin{aligned}
f_{r}(x)-f(x) & =m^{r} \int_{\mathbf{Q}_{m}} \Omega\left(m^{r}|y-x|_{m}\right)(f(y)-f(x)) d_{m} y= \\
= & m^{r} \int_{|y-x|_{m} \leq m^{-r}}(f(y)-f(x)) d_{m} y .
\end{aligned}
$$

Due to continuity of $f(x)$ one gets $\lim _{r \rightarrow \infty}\left(f_{r}(x)-f(x)\right)=0$.

Theorem 4.4. If the functions $f(x)$ and $g(x)$ are continuous and limited on $\mathbf{Q}_{m}$ and $f(x) \in L^{1}\left(\mathbf{Q}_{m}\right), g(x) \in L^{1}\left(\mathbf{Q}_{m}\right)$ then

$$
\int_{\mathbf{Q}_{m}} \chi_{m}(k x)(f * g)(x) d_{m} x=\tilde{f}(k) \tilde{g}(k) .
$$

Proof. Let us apply Theorem 3.1 replacing $f(x)$ and $g(x)$ with $\chi_{m}(k x) f(x)$ and $\chi_{m}(k x) g(x)$ :

$$
\begin{gathered}
\int_{\mathbf{Q}_{m}}\left[\int_{\mathbf{Q}_{m}}\left(\chi_{m}(k y) f(y)\right)\left(\chi_{m}(k(x-y)) g(x-y)\right) d_{m} y\right] d_{m} x= \\
=\int_{\mathbf{Q}_{m}} \chi_{m}(k x) f(x) d_{m} x \int_{\mathbf{Q}_{m}} \chi_{m}(k x) g(x) d_{m} x .
\end{gathered}
$$

Then we obtain

$$
\int_{\mathbf{Q}_{m}} \chi_{m}(k x)\left(\int_{\mathbf{Q}_{m}} f(y) g(x-y) d_{m} y\right) d_{m} x=\int_{\mathbf{Q}_{m}} \chi_{m}(k x) f(x) d_{m} x \int_{\mathbf{Q}_{m}} \chi_{m}(k x) g(x) d_{m} x .
$$

\section{Levi processes with a value in $\mathrm{Q}_{m}$}

According to Kolmogorov's axiomatic [16], by probability space is understood a triplet of the elements $\{\Omega, \Sigma, P\}$, where $\{\Omega, \Sigma\}$ is measurable space, $P$-non-negative countable additive function on $\sigma$-algebra $\Sigma$ satisfying the condition $P(\Omega)=1$. Let $\{\Omega, \Sigma\}$ be measurable space and $\left\{\mathbf{Q}_{m}, \mathrm{~B}\right\}$ is the pair composed of a ring of $m$-adic numbers $\mathbf{Q}_{m}$ and $\sigma$-algebra $\mathrm{B}$ of all measurable subsets of $\mathbf{Q}_{m}$. Mapping $X: \Omega \rightarrow \mathrm{Q}_{m}$ is called $\Sigma \mid \mathrm{B}$ - measurable (or $X \in \Sigma \mid \mathrm{B}$ ) if $X^{-1}(\mathrm{~B}) \subset \Sigma$. $m$ Adic random variable $X=X(\omega)$ is $\Sigma \mid \mathrm{B}$ - measurable mapping $X: \Omega \rightarrow \mathrm{Q}_{m}$. Function $X(\omega)$ generates the probability measure $P_{X}(B)$ on $B \in \mathrm{B}$ by relation $P_{X}(B)=P\left(X^{-1}(B)\right)$. The function $P_{X}(B)$ is called distribution function of $m$-adic random variable $X . X(\omega)$ is continuous, if there exists integrable on any $B \in \mathrm{B}$ function $f(x)$ such that $P_{X}(B)=\int_{B} f(x) d_{m} x$. We shall call function $f(x)$ density of distribution function of $m$-adic random variable $X$. In the simplest case it is 
possible to choose $\Omega \equiv \mathbf{Q}_{m}$ as the probability space $\Sigma \equiv \mathrm{B}, P(B) \equiv \int_{B} f(x) d_{m} x$ for any $B \in \mathrm{B}$ and some function $f(x)$ such that $f(x) \in L_{\mathrm{loc}}^{1}\left(\mathbf{Q}_{m}\right), f(x) \in L^{1}\left(\mathbf{Q}_{m}\right)$ and $\int_{\mathbf{Q}_{m}} f(x) d_{m} x=1$. In such case $X(\omega)=\omega, \omega \in \mathbf{Q}_{m}$, and random variable $X$ is uniquely defined by the density of distribution function $f(x)$.

Random variable $X$ with the values in $\mathbf{Q}_{m}$ is called infinitely divisible if for any $n \geq 1$ it is possible to find independent evenly distributed random variables $X_{n 1}, \ldots, X_{n n}$ with the values in $\mathrm{Q}_{m}$ such that $X=X_{n 1}+\ldots+X_{n n}$.

Characteristic function $\mu(t) m$-adic random variable is defined as

$$
\hat{f}(k)=\int_{\mathbf{Q}_{m}} f(x) \chi(k x) d_{m} x .
$$

Theorem 5.1. Characteristic function of any infinitely divisible m-adic distribution can be represented in the form of (analogous to Levi-Hinchin formula)

$$
\hat{f}(k)=\chi\left(k x_{0}\right) \Omega\left(|k|_{m} p^{-r}\right) \exp \left(\int_{\mathbf{Q}_{m} \backslash\{0\}}(\chi(k x)-1) W(x) d_{m} x\right),
$$

where $x_{0} \in \mathbf{Q}_{m}, r$ is integer, $W(x) d_{m} x$ is the measure on $\mathbf{Q}_{m}$, finite on $\mathbf{Q}_{m} \backslash B_{s}$ for any s, for which $\int_{\mathbf{Q}_{m} \backslash\{0\}}(1-\operatorname{Re\chi }(k x)) W(x) d_{m} x<\infty$ and here $\int_{\mathbf{Q}_{m} \backslash\{0\}} \cdots d_{m} x=$ $\lim _{r \rightarrow-\infty} \int_{\mathbf{Q}_{m} \backslash B_{r}} \cdots d_{m} x$ is the principal value of the integral.

The proof of the Theorem 5.1 coincides with the proof of analogous theorem for totally disconnected locally compact Abel groups (e.g. refer to [19]) also for the field $Q_{p}[20]$.

By $m$-adic number random process we shall call mapping $X=X(t, \omega)$ of $\mathbf{R}_{+} \otimes \Omega$ on $\mathbf{Q}_{m}$ which is $\Sigma \mid$ B-measurable for any $t \in \mathbf{R}_{+}$.

By Levi process $X(t)$ from $\mathbf{Q}_{m}$ we shall call stochastically continuous Markov process with stationary independent increments, beginning from zero and having right continuous trajectories with left side limits, i.e. process for which the following conditions are hold:

1. $X(0)=0$ almost everywhere.

2. For any $0 \leq t_{1}<\ldots<t_{n}$ excess $X\left(t_{1}\right), X\left(t_{2}\right)-X\left(t_{1}\right), \ldots, X\left(t_{n}\right)-X\left(t_{n-1}\right)$ are independent.

3. For any $t$ and $s X(t+s)-X(s)=X(t)-X(0)$.

4. For any $t \geq 0$ and $r \lim _{s \rightarrow t} P\left(|X(s)-X(t)|_{m}>p^{r}\right)=0$.

5. Trajectories $X(t)$ for all $t>0$ almost everywhere right continuous and possess left limits.

From the definition of Levi process it follows that $X(t)$ is infinitely divisible. Characteristic function of Levi process is defined as

$$
\hat{f}(k, t)=\mathrm{M}[\chi(k X(t))]
$$


and due to uniformity and independence of increments has the form

$$
\hat{f}(k, t)=\exp (t \psi(k))
$$

where the form of the function $\psi(k)$ follows from (5.1)

$$
\psi(k)=\int_{\mathbf{Q}_{m} \backslash\{0\}}(\chi(k x)-1) W(x) d_{m} x
$$

It is not difficult to see that $\hat{f}(k, t)=\exp \left(t \int_{\mathbf{Q}_{m} \backslash\{0\}}(\chi(k x)-1) W(x) d_{m} x\right)$ satisfies the equation

$$
\frac{d}{d t} \hat{f}(k, t)=\hat{f}(k, t) \int_{\mathbf{Q}_{m} \backslash\{0\}}(\chi(k x)-1) W(x) d_{m} x .
$$

From (5.2) the equations for the density of distribution function for the Levi process follows

$$
\frac{d}{d t} f(x, t)=\int_{\mathbf{Q}_{m} \backslash\{0\}} W(y-x)(f(y, t)-f(x, t)) d_{m} y
$$

Thus the following theorem takes place.

Theorem 5.2. Distribution function $f(x, t)$ for all Levi process with the values in $\mathbf{Q}_{m}$ satisfies eq. (5.3) where $W(x) d_{m} x$ is the measure on $\mathbf{Q}_{m}$ finite on $\mathbf{Q}_{m} \backslash B_{s}$ for all $s$, for which $\int_{\mathbf{Q}_{m} \backslash\{0\}}(1-\operatorname{Re\chi }(k x)) W(x) d_{m} x<\infty$.

Note that a particular case of Levi process in $\mathbf{Q}_{m}$ is the one described by Vladimirov equation for which $W(x)=-\frac{1}{\Gamma_{m}(-\alpha)} \frac{1}{|x|^{\alpha}}, \Gamma_{m}(-\alpha)=\frac{1-m^{-\alpha-1}}{1-m^{\alpha}}, \alpha>0$ :

$$
\frac{d f(x, t)}{d t}=-\frac{1}{\Gamma_{m}(-\alpha)} \int_{\mathbf{Q}_{m} \backslash\{0\}} \frac{f(y, t)-f(x, t)}{|y-x|_{m}^{\alpha+1}} d_{m} y .
$$

\section{$6 \mathrm{Q}_{m}$-valued uncoupled continuous time random walk}

Let $\left(\xi_{1}, T_{1}\right),\left(\xi_{1}, T_{1}\right), \ldots$ be a sequence of independent identically distributed pairs of random variables $\xi_{i} \in \mathbf{Q}_{m}$ and $T_{i} \in \mathrm{R}_{+}$. Let the distribution of each pair is given by probability measure $P\left(d_{m} x, d t\right)$ on $\mathbf{Q}_{m} \times \mathrm{R}_{+}$. We shall suppose that $\xi_{i}$ and $T_{i}$ are independent and hence $P\left(d_{m} x, d t\right)=\varphi(x) d_{m} x \psi(t) d t$ where $\varphi(x)$ and $\psi(t)$ are probability densities of $\xi_{i}$ and $T_{i}$ respectively. Let us define random process $N(t)$ as

$$
N(t)=\max \left\{n: \sum_{i=1}^{n} T_{i} \leq t\right\}
$$


We shall call the process

$$
X(t)=\sum_{i=1}^{N(t)} \xi_{i}
$$

as $m$-adic uncoupled continuous time random walk ( $m$-adic CTRW).

Let us denote $\sum_{i=1}^{n} T_{i} \equiv t_{n}$. Distribution $p(t, n)$ for $N(t)$ is the measure function $p(t, n)=P(A)$ where $\Sigma \supset A=\left\{\{\omega\}: \forall \omega \in A, t_{n}(\omega)<t \leq t_{n+1}(\omega)\right\}$ or, equivalently as mathematic expectation $p(t, n)=\mathrm{M}\left[I\left(t_{n}<t \leq t_{n+1}\right)\right]$ where

$$
I\left(t_{n}<t \leq t_{n+1}\right)=\left\{\begin{array}{l}
1, t_{n}<t \leq t_{n+1}, \\
0, t_{n} \geq t \text { or } t>t_{n+1} .
\end{array}\right.
$$

Let $\hat{p}(s, n)=L[p(t, n)](s)$ be Laplace image of $p(t, n)$. Then

$$
\hat{p}(s, n)=\mathrm{M}\left[\int_{0}^{\infty} d t e^{-s t} I\left(t_{n}<t<t_{n+1}\right)\right]=\mathrm{M}\left[\frac{e^{-s t_{n+1}}-e^{-s t_{n}}}{s}\right] .
$$

Because $t_{n}=\sum_{i=1}^{n} T_{i}$ is the sum of independent random variables, we have

$$
\mathrm{M}\left[e^{-s t_{n}}\right]=\mathrm{M}\left[\exp \left(-s \sum_{i=1}^{n} T_{i}\right)\right]=\hat{\psi}^{n}(s)
$$

where $\hat{\psi}(s)=L[\psi(t)](s)$. Thus

$$
\hat{p}(s, n)=\mathrm{M}\left[\frac{e^{-s t_{n+1}}-e^{-s t_{n}}}{s}\right]=\hat{\psi}^{n}(s) \frac{1-\hat{\psi}(s)}{s}
$$

Distribution density $f(x, t)$ of a process $X(t)$ is Fourier image of characteristic function of the process $X(t)$

$$
f(x, t)=F^{-1}[\tilde{f}(k, t)] .
$$

Using (6.2), we obtain

$$
\tilde{f}(k, t)=\mathrm{M}\left[\chi\left(k \sum_{i=1}^{N(t)} \xi_{i}\right)\right]=\sum_{i=0}^{\infty} \tilde{\varphi}^{n}(k) p(n, t),
$$

where $\tilde{\varphi}(k)=\mathrm{M}\left[\chi\left(\xi_{i} k\right)\right]$ is characteristic function of independent identically distributed variables $\xi_{i}$. Using (6.3) we have

$$
\hat{\tilde{f}}(k, s)=\frac{1-\hat{\psi}(s)}{s} \frac{1}{1-\hat{\psi}(s) \tilde{\varphi}(k)} .
$$

Formula (6.4) is Monthroll-Weiss equation [21] for $m$-adic CTRW with independent jumps. After simple transformations, the equation (6.4) can be represented in an alternative form

$$
\hat{\Phi}(s)[s \hat{\tilde{f}}(k, s)-1]=[\tilde{\varphi}(k)-1] \hat{\tilde{f}}(k, s),
$$


where

$$
\hat{\Phi}(s)=\frac{1-\hat{\psi}(s)}{s \hat{\psi}(s)} .
$$

Inverting Laplace-Fourier transforms, it is possible to rewrite eq. (6.5) in the following forms:

1. Integral equation

$$
f(x, t)=\Psi(t) \delta(x)+\int_{0}^{t} \psi\left(t-t^{\prime}\right) d t \int_{\mathbf{Q}_{m}} \varphi\left(k-k^{\prime}\right) f(x, t) d_{p} k, \Psi(t)=L^{-1}\left[\frac{1-\hat{\psi}(s)}{s}\right] .
$$

2. Generalized Kolmogorov-Feller equation

$$
\int_{0}^{t} \Phi\left(t-t^{\prime}\right) \frac{d}{d t^{\prime}} f(x, t) d t^{\prime}=\int_{\mathbf{Q}_{m}} d_{p} x^{\prime} \varphi\left(x-x^{\prime}\right)\left(f\left(x^{\prime}, t\right)-f(x, t)\right), \Phi(t)=L^{-1}[\hat{\Phi}(s)] .
$$

For exponential distribution of time intervals between the jumps

$$
\psi(t)=e^{-\lambda t}, \psi(s)=\frac{\lambda}{s+\lambda}, \Phi(t)=\frac{1}{\lambda} \delta(t)
$$

the process (6.2) is Levi process with finite measure function $W(x) d_{m} x \equiv \lambda \varphi(x) d_{m} x$ on the whole $\mathbf{Q}_{m}$.

\section{Diffusive limit of $\mathrm{Q}_{m}$-valued CTRW and rela- tionship with Vladimirov equation}

Let us multiply all waiting times $T_{i}$ by the factor $r$, and $\xi_{i} \in \mathbf{Q}_{m}$ - by the factor $h \in \mathbf{Q}_{m}$. Then the distribution densities $\psi(t)$ and $\varphi(x)$ are transformed into

$$
\psi_{r}(t)=r^{-1} \psi\left(\frac{t}{r}\right), \varphi_{h}(x)=|h|_{p}^{-1} \varphi\left(\frac{x}{h}\right) .
$$

For Laplace and Fourier transforms of $\psi(t)$ and $\varphi(x)$ we have

$$
\hat{\psi}_{r}(s)=\hat{\psi}(r s), \tilde{\varphi}_{h}(k)=\tilde{\varphi}(h k) .
$$

Then Monthroll-Weiss equation (6.4) will have the form

$$
\hat{\tilde{f}}_{r, h}(k, s)=\frac{1-\hat{\psi}_{r}(s)}{s} \frac{1}{1-\hat{\psi}_{r}(s) \tilde{\varphi}_{h}(k)}=\frac{1-\hat{\psi}(r s)}{s} \frac{1}{1-\hat{\psi}(r s) \tilde{\varphi}(h k)}
$$

We propose that $\hat{\psi}(s)$ and $\tilde{\varphi}(k)$ have the following asymptotic behavior at $s \rightarrow 0$, $|k|_{m} \rightarrow 0$ :

$$
\hat{\psi}(s)=1-s^{\beta}+o\left(s^{\beta}\right), 0<\beta \leq 1, \tilde{\varphi}(k)=1-|k|_{m}^{\alpha}+o\left(|k|_{m}^{\alpha}\right), \alpha>0
$$


Then we define diffusive limit of $m$-adic CTRW (similarly to definition of diffusive limit of real value CTRW [22, 23]) as

$$
r \rightarrow 0,|h|_{m} \rightarrow 0 \quad \text { on condition that } r^{\beta}=|h|_{m}^{\alpha}
$$

It is not difficult to see that

$$
\hat{\tilde{f}}_{r, h}(k, s) \rightarrow \hat{\tilde{u}}(k, s) \equiv \frac{1}{s^{\beta}+|k|_{m}^{\alpha}}
$$

In this passage to the limit, $\hat{\tilde{f}}_{r, h}(k, s)$ and $\hat{\tilde{u}}(k, s)$ are asymptotically equivalent in the Laplace-Fourier domain. Then, the asymptotic equivalence in the $\mathbf{Q}_{m}$-space - real time domain between the $f_{r, h}(x, t)$ and $u(x, t) \equiv F^{-1} L^{-1}[\hat{\tilde{u}}(k, s)]$ is ensured by the continuity theorem for sequences of characteristic functions, after the application of the analogous theorem for sequences of Laplace transforms [24]. Exactly in the same way as in the case of diffusive limit of real valued CTRW, it is possible to show that (ref. [22, 23]) that

$$
f_{r, h}(x, t) \rightarrow u(x, t)
$$

Precise evaluations show that

$$
\begin{gathered}
u(x, t)=F^{-1} L^{-1}[\hat{\tilde{u}}(k, s)]=\int_{\mathbf{Q}_{m}} \chi(-k x) E_{\beta}\left(-|k|_{m}^{\alpha} t^{\beta}\right) d_{m} k= \\
=\left(1-m^{-1}\right)|x|_{m}^{-1} \sum_{i=0}^{\infty} m^{-i} E_{\beta}\left(-m^{-i \alpha}|x|_{m}^{-\alpha} t^{\beta}\right)-|x|_{m}^{-1} E_{\beta}\left(-m^{\alpha}|x|_{m}^{-\alpha} t^{\beta}\right),
\end{gathered}
$$

where

$$
E_{\beta}(z)=\sum_{n=0}^{\infty} \frac{z^{n}}{\Gamma(\beta n+1)}
$$

is Mittag-Leffler function. Function (7.1) satisfies the equation

$$
\frac{\partial^{\beta}}{\partial t^{\beta}} u(x, t)=-\frac{1}{\Gamma_{m}(-\alpha)} \int_{\mathbf{Q}_{m}} \frac{u(y, t)-u(x, t)}{|x-y|_{m}^{\alpha+1}} d_{m} y,
$$

where $\frac{\partial^{\beta}}{\partial t^{\beta}}$ is the fraction Caputo derivative [25]

$$
\frac{\partial^{\beta}}{\partial t^{\beta}} f(t) \equiv \frac{1}{\Gamma(1-\beta)}\left(\frac{\partial}{\partial t} \int_{0}^{t} \frac{f(\tau)}{(t-\tau)^{\beta}} d \tau-t^{-\beta} f(0)\right)
$$

We shall call equation (7.2) equation the fractional-time $m$-adic random walk. For $\beta=1$, eq. (7.2) becomes the $m$-adic Vladimirov equation (5.4). 


\section{The equation of fraction-time $m$-adic random walk and asymptotic behavior of the probabil- ity measure of initial distribution support}

We consider the equation of fractional-time $m$-adic random walk (7.2) for $0<\beta \leq$ $1, \alpha>0$ and with the initial condition $u(x, 0)=\Omega\left(|x|_{m} \leq 1\right)$. Solution of eq. (7.2) is easy to find using Laplace-Fourier transformations:

$$
\begin{gathered}
u(x, t)=\Omega\left(|x|_{m} \leq 1\right)\left(1-m^{-1}\right) \sum_{i=0}^{\infty} m^{-r} E_{\beta}\left(-m^{-\alpha i} t^{\beta}\right)+ \\
+\Omega\left(|x|_{m}>1\right)\left(\left(1-m^{-1}\right)|x|_{m}^{-1} \sum_{i=0}^{\infty} m^{-i} E_{\beta}\left(-m^{-\alpha i}|x|_{m}^{-\alpha} t^{\beta}\right)-|x|_{m}^{-1} E_{\beta}\left(-m^{\alpha}|x|_{m}^{-\alpha} t^{\beta}\right)\right) .
\end{gathered}
$$

Probability measure of the support of initial distribution is defined as

$$
S(t)=\int_{Z_{m}} u(x, t) d_{m} x=\left(1-m^{-1}\right) \sum_{i=0}^{\infty} m^{-i} E_{\beta}\left(-m^{-\alpha i} t^{\beta}\right) .
$$

Let us find the estimation of (8.1) at $t \rightarrow \infty$. Note that the function $m^{-x}$ decreases with the increase of $x$, while $E_{\beta}\left(-m^{-\alpha x} t^{\beta}\right)$ increases. Consequently on the segment $i \leq x \leq i+1$ we have in equation

$$
m^{-x} E_{\beta}\left(-m^{-i \alpha(x-1)} t^{\beta}\right) \leq m^{-i} E_{\beta}\left(-m^{-i \alpha} t^{\beta}\right) \leq m^{-(x-1)} E_{\beta}\left(-m^{-\alpha x} t^{\beta}\right) .
$$

Integrating (8.2 over $x$ from $i$ to $i+1$ and then summing by $i$ from 0 to $\infty$, we obtain

$$
\frac{1}{\alpha p \ln p} t^{-\frac{\beta}{\alpha}} \Theta_{\beta}(\alpha, t) \leq S(t) \leq \frac{p}{\alpha \ln p} t^{-\frac{\beta}{\alpha}} \Theta_{\beta}(\alpha, t)
$$

where

$$
\Theta_{\beta}(\alpha, t) \equiv \int_{0}^{t^{\beta}} E_{\beta}(-y) y^{\frac{1}{\alpha}-1} d y, \Theta_{\beta}(\alpha) \equiv \int_{0}^{\infty} E_{\beta}(-y) y^{\frac{1}{\alpha}-1} d y .
$$

Because at $t \rightarrow+\infty$ and $\lambda>0$

$$
E_{\beta}\left(-\lambda t^{\beta}\right)=\frac{\sin (\beta \pi)}{\lambda \pi} \frac{\Gamma(\beta)}{t^{\beta}}(1+o(1)),
$$

the integral in $\Theta_{\beta}(\alpha)$ converges at $\alpha>1$ and we have

$$
\frac{1}{\alpha p \ln p} t^{-\frac{\beta}{\alpha}} \Theta_{\beta}(\alpha)(1+o(1)) \leq S(t) \leq \frac{p}{\alpha \ln p} t^{-\frac{\beta}{\alpha}} \Theta_{\beta}(\alpha)(1+o(1)) .
$$

At $\alpha \leq 1$ when $y \rightarrow+\infty$ we have

$$
E_{\beta}(-y) y^{\frac{1}{\alpha}-1}=\frac{\sin (\beta \pi) \Gamma(\beta)}{\pi} y^{\frac{1}{\alpha}-2}(1+o(1)),
$$


therefore

$$
\int_{0}^{t^{\beta}} E_{\beta}(-y) y^{\frac{1}{\alpha}-1} d y=\left\{\begin{array}{l}
\frac{\sin (\beta \pi) \Gamma(\beta)}{\pi} t^{\beta\left(\frac{1}{\alpha}-1\right)}(1+o(1)), \alpha<1, \\
\frac{\sin (\beta \pi) \Gamma(\beta)}{\pi} \ln t(1+o(1)), \alpha=1 .
\end{array}\right.
$$

Finally we obtain the following asymptotic estimate of $S(t)$

$$
\frac{1}{\alpha p \ln p} R(t) \leq S(t) \leq \frac{p}{\alpha \ln p} R(t)
$$

where at $t \rightarrow+\infty$

$$
R(t)=\left\{\begin{array}{l}
t^{-\frac{\beta}{\alpha}} \Theta_{\beta}(\alpha)(1+o(1)), \alpha>1, \\
\frac{\sin (\beta \pi) \Gamma(\beta)}{\pi} t^{-\beta}(1+o(1)), \alpha<1, \\
\frac{\sin (\beta \pi) \Gamma(\beta)}{\pi} \ln t(1+o(1)), \alpha=1 .
\end{array}\right.
$$

\section{Summary}

In this paper we developed a number of theorems from the $p$-adic [2, 14] into $m$ adic analysis of complex valued function. This allowed us to consider some type of $m$-adic-valued stochastic processes. In particular, the distribution function of any $m$-adic-valued Levy process satisfies the pseudodifferential equation of generalized ultrametric random walk. The class of $m$-adic CTRW random processe was considered and its diffusion limit was obtained. It is well known that diffusion limit of real valued CTRW (fractional-time in general) is the diffusion process. However the trajectories of ultrametric processes are not continuous and any of such process is not diffusion in the traditional definition, because the Lindeberg condition is not hold. But we can define $m$-adic process, being the diffusion limit of $m$-adic CTRW, as diffusion indeed. Nevertheless we do not follow this terminology. We call the diffusion limit of $m$-adic CTRW as fractional-time $m$-adic random walk. The distribution function of such process satisfies the Vladimirov equations with fractional time derivative. We also obtained the fractional-time $m$-adic random walk distributive function in explicit form and check the asymptotic behavior of the probability measure of the initial distribution support at large times.

It is interesting to consider the next generalization of the $m$-adic analysis to polyadic. As it is known [26] for any integers sequence $\ldots, a_{-2}, a_{-1}, a_{0}, a_{1}, a_{2}, \ldots$ there exist the set of polyadic numbers $\mathbf{Q}_{\{a\}}$ that is the set of formal series $x=$ $\cdots+\frac{1}{a_{-3} a_{-2} a_{-1}} x_{-3}+\frac{1}{a_{-2} a_{-1}} x_{-2}+\frac{1}{a_{-1}} x_{-1}+x_{0}+a_{0} x_{1}+a_{1} a_{0} x_{1}+\ldots$, with $x_{i}=$ $0,1, \ldots, a_{i}-1$. The set $\mathbf{Q}_{\{a\}}$ is local compact abelian commutative group and has 
Haar measure and character group, that admits exact realization of measure, Fourier analysis and detailed consideration of polyadic stochastic processes.

\section{Acknowledgements}

A.Z. is grateful to Igor Volovich, Sergey Kozyrev, Vladik Avetisov, Albert Bikulov and Branko Dragovic for useful discussions. Work was partially supported by grants ADTP 3341 and RFBR 09-01-12161-ofi-m.

\section{References}

[1] W.H.Schikhof, Ultrametric Calculus. An Introduction to p-adic Analysis. Cambridge Studies in Advanced Mathematics, 4. Cambridge University Press, Cambridge, 1984. viii+306 pp.

[2] V.S.Vladimirov, I.V.Volovoitch, E.I.Zelenov, p-adic Analysis and Mathematical Physics. - M.: Fizmatlit, 1994, 352 p. Vladimirov, V. S., Volovich, I. V., and Zelenov, E. I. p-Adic Analysis and Mathematical Physics, - Singapore: World Scientific Publishing (1994).

[3] R.Rammal, G.Toulouse, M.A.Virasoro, Ultrametricity for Physicists. Rev. Mod. Phys. 589 (1986) 765.

[4] B.Dragovich, A.Yu.Khrennikov, S.V.Kozyrev, I. V. Volovich, On p-adic mathematical physics. p-Adic Numbers, Ultrametric Analysis, and Applications Vol. 1, No 1, (2009) 1.

[5] A.T.Ogielski, D.L.Stein, Phys. Rev. Lett. 55 (1985) 1634.

[6] V.A.Avetisov, A.Kh.Bikulov, S.V.Kozyrev, V.A.Osipov, p-Adic Models of Ultrametric Diffusion Constrained by Hierarchical Energy Landscapes. J.Phys. A: Math. Gen., 35 (2002) 177.

[7] V.A.Avetisov, A.Kh.Bikulov, S.V.Kozyrev, Application of p-adic Analysis to Models of Spontaneous Breaking of Replica Symmetry. Journal of Physics A, 1999. Vol.32, p. 8785.

[8] V.A.Avetisov, A.Kh.Bikulov, V.A.Osipov, p-Adic Description of Characteristic Relaxation in Complex Systems. J. Phys. A: Math. Gen. 36, 2003, 4239.

[9] V.A.Avetisov, A.Kh.Bikulov, A.P.Zubarev, First Passage Time Distribution and the Number of Returns for Ultrametric Random Walks. J. Phys. A: Math. Theor. 42, (2009) p. $085003-085020$.

[10] R.N.Mantenga, Hierarchical Structure in Financial Market. Eur. Phys. J. B 11 (1999) 193.

[11] R.N.Mantenga, H.E.Stanley , An Introduction to Econophysics. Correlations and Complexity in Finance, Cambridge Univ. Press, Cambridge, 2000. 
[12] A.Kh.Bikulov, A.P.Zubarev, L.V.Kaidalova, Hierarchic Dynamic Model of Financial Market near Crash Points and $p$-adic Mathematical Analysis. Proc. Samara State Tech. Univ., Iss. 42, 2006, p. 135.

[13] S.V.Kozyrev, A.Yu.Khrennikov, Pseudodifferential Operators on Ultrametric Spaces and Ultrametric Wavelets, Izv. RAN. Ser. Mat., 69:5 (2005), p. 133148.

[14] V.S.Vladimirov, Tables of Integrals of Complex-Valued Functions of $p$-adic Arguments. Sovrem. Probl. Mat., 2, Steklov Math. Inst., RAS, Moscow, 2003, p. $3-88$.

[15] A.Yu.Khrennikov, Non-Archimedean analysis: Quantum Paradoxes, Dynamical Systems and Biological Models. Kluwer Acad. Publishers, Dordreht/Boston/London, 1997.

[16] A.N.Kolmogorov, S.V. Fomin, Introduction to the Theory of Functions and to Functional Analysis. M.: Nauka, 1954 (in Russian).

[17] G.E.Shilov, Mathematical Analysis M.: Nauka, 1979, vol. 1, 2, 3 (in Russian).

[18] A.N.Shiryaev, Probability. 2nd ed., Nauka, Moscow, 1989.

[19] K.R.Parthasarathy, Probability Measure on Metric Spaces. Academic Press Inc., London, 1967.

[20] A.N.Kochubei, Pseudo-Differential Equations and Stochastics over NonArchimedean Fields. Marcel Dekker, New York, 2001.

[21] E.W.Montroll, G.H.Weiss, J. Math. Phys. 6, 167 (1965).

[22] R.Gorenflo, F.Mainardi, Continuous Time Random Walk, Mittag-Leffler Waiting Time and Fractional Diffusion: Mathematical Aspects, Chap. 4. In: R. Klages, G. Radons, and I.M. Sokolov (Editors): "Anomalous Transport: Foundations and Applications", Wiley-VCH, Weinheim, Germany, 2008, pp. 93-127.

[23] E.Scalas, R.Gorenflo, F.Mainardi, Uncoupled Continuous-Time Random Walks: Solution and Limiting Behavior of the Master Equation. Phys Rev E Stat Nonlin Soft Matter Phys. 2004 Jan; 69 (1 Pt 1): 011107.

[24] W.Feller, An Introduction to Probability Theory and its Applications, Vol. II. Second Edition. Wiley, New York, 1971.

[25] M.Caputo, F.Mainardi, Riv. Nuovo Cimento (Ser. II), 1971, 1, 161-198.

[26] E.Hewitt, K.A.Ross, Abstract Harmonic Analysis. Springer-Verlag, 1987. 


\title{
Some aspects of the $\boldsymbol{m}$-adic analysis and its applications to $\boldsymbol{m}$-adic stochastic processes
}

\author{
M.V. Dolgopolov, * \\ and
}

A.P. Zubarev ${ }^{\dagger}$

November 8, 2018

\begin{abstract}
In this paper we consider a generalization of analysis on $p$-adic numbers field to the $m$ case of $m$-adic numbers ring. The basic statements, theorems and formulas of $p$-adic analysis can be used for the case of $m$-adic analysis without changing. We discuss basic properties of $m$-adic numbers and consider some properties of $m$-adic integration and $m$-adic Fourier analysis. The class of infinitely divisible $m$-adic distributions and the class of $m$-adic stochastic Levi processes were introduced. The special class of $m$-adic CTRW process and fractional-time $m$-adic random walk as the diffusive limit of it is considered. We found the asymptotic behavior of the probability measure of initial distribution support for fractional-time $m$-adic random walk.
\end{abstract}

\section{Introduction}

Up to recent time, ultrametric spaces were known only in mathematical literature. A classic example of such space is the $p$-adic numbers [1, 2] field. During the last three decades the interest in ultrametric models research has risen in various fields of physics, biology, economics and sociology, such as models of spin glass, biopolymers, fractal structures as well as in optimization theory, taxonomy, evolutional biology, cluster and factor analysis etc. (refer to the surveys [3] and [4). In recent years the scientific interest laid also in the field of the dynamic models for the ultrametric structures, in particular the models of ultrametric diffusion (see [5, 6, 7, 8, 9] and references therein), which are directly related to the description of the conformational rearrangements of the proteins dynamics as well, apparently may be related

\footnotetext{
*Samara State University, e-mail: mikhaildolgopolov@rambler.ru
}

${ }^{\dagger}$ Samara State University, e-mail: apzubarev@mail.ru 
to the random processes modeling in the complicated biological and socio-economic systems [10, 11, 12].

The ultrametricity of space is closely related to a concept of hierarchic structure over space [3]. For complex systems modeling hierarchic structures may appear both on the space of states of the system and on the space of objects constituting the system. E. g., one describes dynamics of conformation rearrangements of biopolymer macromolecules, multiple local minima of free energy can be regarded as highly degenerated space of the system states possessing the hierarchic structure [5, 6, 7, 8].

An adequate mathematical tools to formalize description of mathematical modeling of the complex systems hierarchic structures are required. One such tool is a $p$-adic analysis developed during the last three decades by the group of Academician V.S. Vladimirov in Steklov Mathematical Institute. The main idea of application of $p$-adic analysis at mathematical modeling of complex hierarchically organized systems is that the field of $p$-adic numbers $\mathbf{Q}_{p}$ has a natural regular indexed hierarchic structure with the degree of regularity equal to certain prime number $p$ and thus a configuration space of such systems can be adequately described by $p$-adic coordinate space.

Nevertheless, it is worth to mention that, strictly speaking, the mathematical tools of $p$-adic analysis is directly applicable for describing a hierarchically organized system only in the case when the system possesses regular indexed hierarchic structure and degree of regularity of the hierarchic structure is a prime number $p$. The analysis on general ultrametric spaces described by oriented trees has been developed in [13]. Nevertheless, for specific computations it is necessary to use explicit parametrization of the points of an ultrametric space, in which the computational results are represented. For example, in the case of $\mathbf{Q}_{p}$ such parametrization has the form of $a_{n} p^{n}+a_{n-1} p^{n-1}+\ldots$. The using of such a parametrization for ultrametric space corresponding to an oriented tree of general type is rather difficult.

In this paper, which is partly methodological by nature, we consider a generalization an analysis on a field $\mathbf{Q}_{p}$ for more general case of ring of $m$-adic numbers $\mathbf{Q}_{m}$. Some aspects of $m$-adic analysis are considered in the book [14]. The ring $\mathbf{Q}_{m}$ as well as the field $\mathbf{Q}_{p}$ is local-compact Abelian group. Thus for $\mathbf{Q}_{m}$ there exists a unique (up to a normalization) translation-invariant Haar measure and dual group of characters which allows to develop Fourier analysis and consider the classes of pseudo-differential operators. We show that the basic statements, theorems and formulas of $p$-adic analysis [2, 15] can be used for the case of $m$-adic analysis without changing. In section 2 we discuss basic properties of $m$-adic numbers $\mathbf{Q}_{m}$. In sections 3 and 4 we consider some properties of $m$-adic integration and $m$-adic Fourier analysis. In section 5 we introduce a class of infinitely divisible $m$-adic distributions and a class of $m$-adic random Levi processes. In section 6 we consider the special class of random processes of uncoupled continuous time random walk type on $\mathbf{Q}_{m}$ ( $m$-adic CTRW). In section 7 we discuss the diffusive limit of $m$-adic CTRW together with the equation of fraction-time $m$-adic random walk. In section 8 we find the asymptotic behavior of probability measure of initial distribution support for a random process described by fraction-time equation of $m$-adic random walk. 


\section{The ring of $m$-adic numbers $\mathrm{Q}_{m}$}

Let us define a ring $\mathbf{Q}_{m}$ as the set of infinite series

$$
x=\sum_{i=r}^{\infty} a_{i} m^{i},
$$

where $r$ is an integer number, $a_{r}=1,2,3, \ldots, m-1, a_{i}=0,1,2,3, \ldots, m-1(i \neq r)$. The set $\mathbf{Q}_{m}$ has a structure of commutative associative ring, i.e. the sum and product operations are defined in it. Let us call a sum of two $m$-adic numbers $x=\sum_{i=r}^{\infty} a_{i} m^{i}$ and $y=\sum_{i=s}^{\infty} b_{i} m^{i}$ such element $z=\sum_{i=l}^{\infty} c_{i} m^{i}, l=\min \{r, s\}$ that

$$
\sum_{i=r}^{\infty} a_{i} m^{i}+\sum_{i=s}^{\infty} b_{i} m^{i}=\sum_{i=l}^{\infty} c_{i} m^{i} .
$$

Let us call the product of two $m$-adic numbers $x=\sum_{i=r}^{\infty} a_{i} m^{i}$ and $y=\sum_{i=s}^{\infty} b_{i} m^{i}$ such an element $z=\sum_{i=l}^{\infty} c_{i} m^{i}$ that $l \geq r+s$ and

$$
\sum_{i=r}^{\infty} \sum_{j=s}^{\infty} a_{i} b_{j} m^{i} m^{j}=\sum_{i=r+s}^{\infty} c_{i} m^{i} .
$$

Comparing right and left parts of equations (2.1) and (2.2) by $\bmod m^{n}$ for $n=$ $1,2,3, \ldots$ makes it possible to find successively the coefficients $c_{i}$ for the sum $z=x+y$ and product $z=x y$.

Division operation in $\mathbf{Q}_{m}$ is uniquely defined only in case $m$ is a prime $p$. In such case, the ring $\mathbf{Q}_{m}$ is a field of $p$-adic numbers $\mathbf{Q}_{p}$.

There exists $m$-adic pseudonorm $|x|_{m}=m^{-r}$ in $\mathbf{Q}_{m}$ for $x=\sum_{i=r}^{\infty} a_{i} m^{i}$ with $a_{r} \neq 0$ which satisfies the following properties:

1. $|x|_{m}=0 \Leftrightarrow x=0$,

2. $|x y|_{m} \leq|x|_{m}|y|_{m}$,

3. $|x+y|_{m} \leq \max \left\{|x|_{m},|y|_{m}\right\}$.

In the case $m$ is a prime $p$, non-eq. 2. transforms into eq. $|x y|_{p}=|x|_{p}|y|_{p}$, and $m$ adic pseudonorm $|x|_{m}$ transforms into $p$-adic norm $|x|_{p}$. Note that for any $x \in \mathbf{Q}_{m}$ and any $r \in \mathbf{Z}$, the following equation takes place:

$$
\left|m^{r} x\right|_{m}=m^{-r}|x|_{m}
$$

Pseudonorm $|x|_{m}$ induces metrics $d_{m}(x, y)=|x-y|_{m}$ satisfying the following properties:

1. $d_{m}(x, y)=0$ then and only then if $x=y$,

2. $d_{p}(x, y) \leq \max \left\{d_{p}(x, z), d_{p}(y, z)\right\}$ for any $x, y$ and $z$ from $\mathbf{Q}_{m}$. 
We call the subset $B_{r}\left(x_{0}\right)=\left\{x \in \mathbf{Q}_{m}:\left|x-x_{0}\right| \leq m^{r}\right\}$ a disk of radius $m^{r}$ with the center at the point $x_{0} \in \mathbf{Q}_{m}$, and subset $S_{r}\left(x_{0}\right)=\left\{x \in \mathbf{Q}_{m}:\left|x-x_{0}\right|=m^{r}\right\}-$ a circumference of radius $m^{r}$ with the center at the point $x_{0} \in \mathbf{Q}_{m}$. Also we denote $B_{r}(0) \equiv B_{r}, S_{r}(0) \equiv S_{r}, B_{0} \equiv Z_{m}$. Due to the same metric structure of both $\mathbf{Q}_{m}$ and $\mathbf{Q}_{p}$ the following statements for $\mathbf{Q}_{m}$ take place [2]:

1. All disks and circumferences in $\mathbf{Q}_{m}$ are closed and open sets.

2. All points of a disk is also its center.

3. If two disks have a common point, then one of them is contained in another.

4. All sets open in $\mathbf{Q}_{m}$ are countable unions of nonintersecting disks.

5. $\mathrm{Q}_{m}$ has zero dimension.

6. $\mathbf{Q}_{m}$ is separable totally disconnected, Hausdorf, locally compact.

\section{Measure and integration on $\mathrm{Q}_{m}$}

As $\mathbf{Q}_{m}$ is a local compact commutative group there exist an additive translation invariant measure $\mu\left(B \in \mathbf{Q}_{m}\right)[2$. This measure is uniquely determined by properties:

1. $\mu\left(Z_{m}\right)=1$ (normalization),

2. $\forall a \in \mathbf{Q}_{m}, \forall B \in \mathrm{B} \mu(B)=\mu(B+a)$ (translational invariance),

3. $\forall\left\{B_{i} \in \mathrm{B}\right\}, B_{i} \cap B_{j}=\emptyset, i \neq j \Rightarrow \mu\left(\bigcup_{i} B_{i}\right)=\sum_{i} \mu\left(B_{i}\right)$ (additivity).

Note that the measure satisfies the following property $\mu\left(m^{r} A\right)=m^{-r} \mu(A)$ but in general $\mu(x A) \neq|x|_{m} \mu(A)$. For example in the case $m=4$ we have $2 \cdot B_{0} \subset B_{0}$. On the other hand

$$
\begin{gathered}
2 \cdot B_{0}=2 \cdot\left(\left(0+B_{-1}\right) \bigcup\left(1+B_{-1}\right) \bigcup\left(2+B_{-1}\right) \bigcup\left(3+B_{-1}\right)\right)= \\
=\left(2 \cdot B_{-1}\right) \bigcup\left(2+2 \cdot B_{-1}\right) \bigcup\left(4+2 \cdot B_{-2}\right) \bigcup\left(2+4+B_{-1}\right),
\end{gathered}
$$

therefore $3+B_{-1} \not \subset 2 \cdot B_{0}$ and $2 \cdot B_{0} \subset B_{0} \backslash\left(3+B_{-1}\right)$. By additivity $\mu\left(2 \cdot B_{0}\right) \leq$ $\mu\left(B_{0}-\left(3+B_{-1}\right)\right)=\mu\left(B_{0}\right) \backslash \mu\left(3+B_{r-1}\right)=4^{r}-4^{r-1}$ and $\mu\left(2 \cdot B_{r}\right) \neq|2|_{4} \mu\left(B_{r}\right)=$ $4^{r}$.

For $B \subset \mathbf{Q}_{m}$ let us call

$$
\mu^{\star}(B)=\inf _{B \subset \cup B_{i}} \sum_{i} \mu_{i}\left(B_{i}\right)
$$

an external measure, where the infimum is taken over all possible coverings of the $B$ by countable set of non-intersecting disks $\left\{B_{i}\right\} . B$ is measurable if for any $\varepsilon>0$ it is possible to find such elementary set (finite union of non-intersecting disks) $A$ that $\mu^{\star}((B \bigcup A) \backslash(B \bigcap A))<\varepsilon$. Let B be the class of all measurable subsets of $\mathbf{Q}_{m}$. 
Then for any $B \in \mathrm{B} \mu(B) \equiv \mu^{*}(B)$. It is possible to prove (e.g. refer to [16]) that $\mathrm{B}$ is $\sigma$-algebra and the measure $\mu(B)$ is $\sigma$-additive on $\mathrm{B}$.

A complex valued function $f(x)$ on $B \in \mathrm{B}$ is measurable if for any Borelean $\mathrm{B} \subset \mathbf{C}$ the set $f^{-1}(\mathrm{~B})$ is measurable one. For measurable functions $f(x)$ it is possible to define linear continuous functional - integral $\int_{B} f(x) d_{m} x$ of the function $f(x)$ over the measure $\mu(B)$.

The set of measurable on $B$ functions for which the integral $\int_{B}|f(x)|^{\lambda} d_{m} x$ at $\lambda \geq 1$ is finite, will be denoted by $L^{\lambda}(B)$. Let $f(x) \in L_{\text {loc }}^{\lambda}\left(\mathbf{Q}_{m}\right)$ if for any measurable compact set $B \in \mathbf{Q}_{m} f(x) \in L^{\lambda}(B)$.

A convolution $(f * g)(x)$ of two functions is

$$
(f * g)(x)=\int_{\mathbf{Q}_{m}} f(y) g(x-y) d_{m} y .
$$

Theorem 3.1. Let the functions $f(x)$ and $g(x)$ are continuous and bounded on $\mathbf{Q}_{m}, f(x) \in L^{1}\left(\mathbf{Q}_{m}\right), g(x) \in L^{1}\left(\mathbf{Q}_{m}\right)$. Then $(f * g)(x)$ exists for any $x$. Moreower, $(f * g)(x)$ is continuous and bounded, $(f * g)(x) \in L^{1}\left(\mathbf{Q}_{m}\right)$ and

$$
\int_{\mathbf{Q}_{m}}(f * g)(x) d_{m} x=\int_{\mathbf{Q}_{m}} f(x) d_{m} x \int_{\mathbf{Q}_{m}} g(x) d_{m} x .
$$

The proof is the same as in regular analysis (e.g. see [17]).

Below some integrals, that we need later, are present.

If a function $f(x)$ depends only on $|x-a|_{m}$, then the integral of it over disk $B_{r}(a)$ equals to

$$
\int_{B_{r}(a)} f\left(|x-a|_{m}\right) d_{m} x=\left(1-m^{-1}\right) \sum_{i=-\infty}^{r} m^{i} f\left(m^{i}\right) .
$$

Let us define the function

$$
\chi_{m}(x)=\exp (2 \pi i\{x\}),
$$

where $\{x\}$ is fraction part of $x$ defined as

$$
\left\{\sum_{i=r}^{\infty} a_{i} m^{i}\right\}=\left\{\begin{array}{l}
\sum_{i=r}^{-1} a_{i} m^{i}, r<0 \\
0, r \geq 0
\end{array}\right.
$$

Let us evaluate the integral $\int_{B_{r}} \chi_{m}(k x) d_{m} x$ where $k \in \mathbf{Q}_{m}$. For $|k|_{m} \leq m^{-r}$ the following relations $|k x|_{m} \leq|k|_{m}|x|_{m} \leq 1$ and $\chi_{m}(k x)=1$ take place, therefore we have $\int_{B_{r}} \chi_{m}(k x) d_{m} x=m^{r}$. At $|k|_{m}>m^{-r}$ we shall make a substitution $x \rightarrow y=x-m^{r}$, $B_{r} \rightarrow B_{r}\left(p^{r}\right)=B_{r}$. Then

$$
\int_{B_{r}} \chi_{m}(k x) d_{m} x=\int_{B_{r}} \chi_{m}\left(k y+k m^{r}\right) d_{m} y=\chi_{m}\left(k m^{r}\right) \int_{B_{r}} \chi_{m}(k y) d_{m} y
$$


and we obtain

$$
\left(1-\chi_{m}\left(k m^{r}\right)\right) \int_{B_{r}} \chi_{m}(k y) d_{m} y=0 .
$$

As $|k|_{m}>m^{-r}($ see (2.3) $)$ we have $\left|k m^{r}\right|_{m}=|k|_{m}\left|m^{r}\right|_{m}=|k|_{m} m^{-r}>1$. By (3.2) (3.3) $\left(1-\chi_{m}\left(k^{r}\right)\right) \neq 0$ it follows from (3.4) that $\int_{B_{r}} \chi_{m}(k y) d_{m} y=0$. Therefore we have

$$
\int_{B_{r}} \chi_{m}(k y) d_{m} y=\left\{\begin{array}{l}
m^{r},|k|_{m} \leq m^{-r}, \\
0,|k|_{m}>m^{-r} .
\end{array}\right.
$$

From (3.5) it follows that

$$
\int_{S_{r}} \chi_{m}(k y) d_{m} y=\left\{\begin{array}{l}
m^{r}\left(1-m^{-1}\right),|k|_{m} \leq m^{-r} \\
-m^{r-1},|k|_{m}=m^{-r+1}, \\
0,\left.k\right|_{m}>m^{-r+1} .
\end{array}\right.
$$

It is not difficult to find for any $f\left(|x|_{m}\right) \in L_{\mathrm{loc}}^{1}\left(\mathbf{Q}_{m}\right)$

$$
\begin{gathered}
\int_{B_{r}} f\left(|x|_{m}\right) \chi_{m}(k x) d_{m} x= \\
=\left\{\begin{array}{c}
\left(1-m^{-1}\right) \sum_{r=-s}^{\infty} m^{-r} f\left(m^{-r}\right),|k|_{m} \leq m^{-s}, \\
\left(1-m^{-1}\right) \frac{1}{|k|_{m}} \sum_{r=0}^{\infty} m^{-r} f\left(\frac{m^{-r}}{|k|_{m}}\right)-\frac{1}{|k|_{m}} f\left(\frac{m}{|k|_{m}}\right),|k|_{m}>m^{-s} .
\end{array}\right.
\end{gathered}
$$

Note that the integrals (3.1), (3.5), (3.6) and (3.7) coincide with corresponding integrals on $\mathbf{Q}_{p}[2$ after substitution $p \rightarrow m$.

\section{Fourier analysis on $\mathrm{Q}_{m}$}

The character of $\mathbf{Q}_{m}$ as an abelian additive group is a complex-valued function $\chi(x)$ on $\mathbf{Q}_{m}$ with the following properties:

1. $|\chi(x)|=1$,

2. $\chi(x)=1 \Leftrightarrow x=0$,

3. $\forall x, y \in Q_{m} \chi(x+y)=\chi(x) \chi(y)$.

Theorem 4.1. The group of characters on group $\mathbf{Q}_{m}$ is isomorphous to $\mathbf{Q}_{m}$, and arbitrary element $\chi(x)$ from group of characters on group $\mathbf{Q}_{m}$ is given by $\chi_{m}(k x)=$ $\exp (2 \pi i\{k x\}), k \in \mathbf{Q}_{m}$. 
Proof. Consider some character $\chi(x)$. Let $\int_{B_{r}} \chi(x) d_{m} x=c$. If $c \neq 0$, then for all $y \in B_{r}$ from the equation

$$
\int_{B_{r}} \chi\left(x^{\prime}\right) d_{m} x^{\prime}=\int_{B_{r}} \chi(x+y) d_{m}(x+y)=\chi(y) \int_{B_{r}} \chi(x) d_{m} x
$$

it follows that $\chi(y)=1$. Let $s=\max \left\{r: \int_{B_{r}} \chi(x) d_{m} x \neq 0\right\}$. Note that $s$ exists, if $\chi(x) \neq 1 \forall x$. Consider arbitrary $x \in \mathbf{Q}_{m}$

$$
x=m^{-r}\left(b_{0}+m b_{1}+m^{2} b_{2}+\ldots+m^{r-s-1} b_{r-s-1}\right)+m^{-s}\left(a_{0}+m a_{1}+m^{2} a_{2}+\ldots\right)
$$

As $\chi\left(B_{s}\right)=1$ we get

$$
\chi(x)=\left(\chi\left(m^{-r}\right)\right)^{b_{0}}\left(\chi\left(m^{-r+1}\right)\right)^{b_{1}} \ldots\left(\chi\left(m^{-s-1}\right)\right)^{b_{r-s-1}}
$$

After some transformations

$$
\begin{gathered}
\chi\left(m^{-r}\right)=\chi\left(m^{-s+s-r}\right)=\left(\chi\left(m^{-s}\right)\right)^{m^{s-r}}=\exp \left(2 \pi i\left(m^{-r+s} l\right)\right), \\
\chi\left(m^{-r+1}\right)=\chi\left(m^{-r}\right)^{m}=\exp \left(2 \pi i\left(m^{-r+s+1} l\right)\right) \\
\cdots \\
\chi\left(m^{-s-1}\right)=\left(\chi\left(m^{-r}\right)\right)^{m^{r-s-1}}=\exp \left(2 \pi i\left(m^{-1} l\right)\right),
\end{gathered}
$$

where $l=0,1, \ldots, m^{r-s}-1$, we get

$$
\chi(x)=\exp \left(2 \pi i\left(\left(l m^{s}\right)\left(m^{-r} b_{0}+m^{-r+1} b_{1}+\ldots+m^{-s-1} b_{r-s-1}\right)\right)\right) .
$$

By denoting $l m^{s} \equiv k$ and expanding $k=m^{s}\left(k_{0}+m k_{1}+\ldots+m^{r-s-1} k_{r-s-1}\right)$ we have

$$
\begin{gathered}
\chi(x)= \\
=\exp \left[2 \pi i \left(\left(m^{s}\left(k_{0}+m k_{1}+\ldots+m^{r-s-1} k_{r-s-1}\right)\right) \times\right.\right. \\
\left.\left.\left(m^{-r}\left(b_{0}+m b_{1}+\ldots+m^{r-s-1} b_{r-s-1}\right)\right)\right)\right] .
\end{gathered}
$$

Thus every character for $\forall x \in \mathbf{Q}_{m}$ is defined by $k \in \mathbf{Q}_{m}$ and has the form

$$
\chi(x)=\exp (2 \pi i k x)=\exp (2 \pi i\{k x\}) .
$$

Let $f(x) \in L^{1}\left(\mathbf{Q}_{m}\right)$, then for any $k \in \mathbf{Q}_{m} f(x) \chi_{m}(k x) \in L^{1}\left(\mathbf{Q}_{m}\right)$. Let us call $m$-adic Fourier transform of a function $f(x)$ such function $\tilde{f}(k)$ that

$$
\tilde{f}(k)=\int_{\mathbf{Q}_{m}} \chi_{m}(k x) f(x) d_{m} x .
$$

Function $f(x)$ on $\mathbf{Q}_{m}$ is called locally constant if $f(x)$ is finite for all $x \in \mathbf{Q}_{m}$, and for all $x \in \mathbf{Q}_{m}$ there exists integer $l(x)$ that $f\left(x+x^{\prime}\right)=f(x)$ for any $x^{\prime} \in B_{l}(x)$. If there exists integer $l$ such that for all $x \in \mathbf{Q}_{m}$ and for all $x^{\prime} \in B_{l}$ the equation 
$f\left(x+x^{\prime}\right)=f(x)$ holds, then $f(x)$ is called locally constant on $\mathbf{Q}_{m}$ with constancy parameter $l$. Let us implement the following designations:

$E\left(\mathbf{Q}_{m}\right)$ is set of functions locally constant on $\mathbf{Q}_{m}$;

$E^{l}\left(\mathbf{Q}_{m}\right)$ is set of functions locally constant on $\mathbf{Q}_{m}$ with constancy parameter $l$;

$D\left(\mathbf{Q}_{m}\right)$ is set of functions on $\mathbf{Q}_{m}$ with compact support;

$D_{r}\left(\mathbf{Q}_{m}\right)$ is set of functions on $\mathbf{Q}_{m}$ with a support in a disk $B_{r}$;

$D_{r}^{l}\left(\mathbf{Q}_{m}\right)$ is set of functions locally constant on $\mathbf{Q}_{m}$ with support in disk $B_{r}$ and constancy parameter $l$.

Theorem 4.2. If $f(x) \in D_{r}^{l}\left(\mathbf{Q}_{m}\right)$, then $\tilde{f}(k) \in D_{-l}^{-r}\left(\mathbf{Q}_{m}\right)$.

Proof. Let $k^{\prime} \in B_{-r}$. Then

$$
\tilde{f}\left(k+k^{\prime}\right)=\int_{\mathbf{Q}_{m}} \chi_{m}\left(k x+k^{\prime} x\right) f(x) d_{m} x=\int_{B_{r}} \chi_{m}(k x) \chi_{m}\left(k^{\prime} x\right) f(x) d_{m} x
$$

Because in the integrand $\left|k^{\prime} x\right|_{m} \leq\left|k^{\prime}\right|_{m}|x|_{m} \leq 1$, then $\chi_{m}\left(k^{\prime} x\right)=1$, we have $\tilde{f}(k+$ $\left.k^{\prime}\right)=\int_{B_{r}} \chi_{m}(k x) f(x) d_{m} x=\int_{\mathbf{Q}_{m}} \chi_{m}(k x) f(x) d_{m} x=\tilde{f}(k)$ and $\tilde{f}(k) \in E^{-r}\left(\mathbf{Q}_{m}\right)$. Let $|k|_{m}>m^{-l}$. Then after substitution $x \rightarrow y=x-m^{-l}$ we get

$$
\begin{gathered}
\tilde{f}(k)=\int_{\mathbf{Q}_{m}} \chi_{m}(k x) f(x) d_{m} x=\int_{\mathbf{Q}_{m}} \chi_{m}\left(k y+k m^{-l}\right) f\left(y+m^{-l}\right) d_{m} y= \\
=\chi_{m}\left(k m^{-l}\right) \int_{\mathbf{Q}_{m}} \chi_{m}(k y) f(y) d_{m} y=\chi_{m}\left(k m^{-l}\right) \tilde{f}(k) .
\end{gathered}
$$

Because $\left|k m^{-l}\right|_{m}=|k|_{m}\left|m^{-l}\right|_{m}=|k|_{m} m^{l}>1$, we have $\left(1-\chi_{m}\left(k m^{-l}\right)\right) \neq 0$ hence $\tilde{f}(k)=0$ and $\tilde{f}(k) \in D_{-l}^{-r}\left(\mathbf{Q}_{m}\right)$.

Theorem 4.3. Let a function $f(x)$ be continuous on $\mathbf{Q}_{m}$ and $f(x) \in L^{1}\left(\mathbf{Q}_{m}\right)$. Then the inversion formula holds:

$$
f(x)=\int_{\mathbf{Q}_{m}} \chi_{m}(-k x) \tilde{f}(k) d_{m} k .
$$

Proof. Consider

$$
f_{r}(x) \equiv \int_{B_{r}} \chi_{m}(-k x)\left(\int_{\mathbf{Q}_{m}} \chi_{m}(k y) f(y) d_{m} y\right) d_{m} k .
$$

Let us show that $\lim _{r \rightarrow \infty} f_{r}(x)=f(x)$. By uniform convergence of the internal integral we have

$$
\begin{aligned}
f_{r}(x) & =\int_{\mathbf{Q}_{m}} \int_{B_{r}} \chi_{m}(k(y-x)) d_{m} k f(y) d_{m} y= \\
& =m^{r} \int_{\mathbf{Q}_{m}} \Omega\left(m^{r}|y-x|_{m}\right) f(y) d_{m} y,
\end{aligned}
$$


where $\Omega(\lambda)=\left\{\begin{array}{l}1, \lambda \leq 1, \\ 0, \lambda>1 .\end{array}\right.$ Taking into account $m^{-r} \int_{\mathbf{Q}_{m}} \Omega\left(m^{r}|y-x|_{m}\right) d_{m} y=1$, we have

$$
\begin{aligned}
f_{r}(x)-f(x) & =m^{r} \int_{\mathbf{Q}_{m}} \Omega\left(m^{r}|y-x|_{m}\right)(f(y)-f(x)) d_{m} y= \\
= & m^{r} \int_{|y-x|_{m} \leq m^{-r}}(f(y)-f(x)) d_{m} y .
\end{aligned}
$$

Due to continuity of $f(x)$ one gets $\lim _{r \rightarrow \infty}\left(f_{r}(x)-f(x)\right)=0$.

Theorem 4.4. If the functions $f(x)$ and $g(x)$ are continuous and bounded on $\mathbf{Q}_{m}$ and $f(x) \in L^{1}\left(\mathbf{Q}_{m}\right), g(x) \in L^{1}\left(\mathbf{Q}_{m}\right)$ then

$$
\int_{\mathbf{Q}_{m}} \chi_{m}(k x)(f * g)(x) d_{m} x=\tilde{f}(k) \tilde{g}(k) .
$$

Proof. Let us apply Theorem 3.1 replacing $f(x)$ and $g(x)$ with $\chi_{m}(k x) f(x)$ and $\chi_{m}(k x) g(x)$ :

$$
\begin{gathered}
\int_{\mathbf{Q}_{m}}\left[\int_{\mathbf{Q}_{m}}\left(\chi_{m}(k y) f(y)\right)\left(\chi_{m}(k(x-y)) g(x-y)\right) d_{m} y\right] d_{m} x= \\
=\int_{\mathbf{Q}_{m}} \chi_{m}(k x) f(x) d_{m} x \int_{\mathbf{Q}_{m}} \chi_{m}(k x) g(x) d_{m} x .
\end{gathered}
$$

Then we obtain

$$
\int_{\mathbf{Q}_{m}} \chi_{m}(k x)\left(\int_{\mathbf{Q}_{m}} f(y) g(x-y) d_{m} y\right) d_{m} x=\int_{\mathbf{Q}_{m}} \chi_{m}(k x) f(x) d_{m} x \int_{\mathbf{Q}_{m}} \chi_{m}(k x) g(x) d_{m} x .
$$

\section{Levi processes with a value in $\mathrm{Q}_{m}$}

According to Kolmogorov's axiomatic [18], by probability space is understood a triplet of the elements $\{\Omega, \Sigma, P\}$, where $\{\Omega, \Sigma\}$ is measurable space, $P$ - non-negative countable additive function on $\sigma$-algebra $\Sigma$ satisfying the condition $P(\Omega)=1$. Let $\{\Omega, \Sigma\}$ be measurable space and $\left\{\mathbf{Q}_{m}, \mathrm{~B}\right\}$ is the pair composed of a ring of $m$-adic numbers $\mathbf{Q}_{m}$ and $\sigma$-algebra $\mathrm{B}$ of all measurable subsets of $\mathbf{Q}_{m}$. Mapping $X: \Omega \rightarrow \mathrm{Q}_{m}$ is called $\Sigma \mid \mathrm{B}$ - measurable (or $X \in \Sigma \mid \mathrm{B}$ ) if $X^{-1}(\mathrm{~B}) \subset \Sigma$. $m$ Adic random variable $X=X(\omega)$ is $\Sigma \mid \mathrm{B}$ - measurable mapping $X: \Omega \rightarrow \mathrm{Q}_{m}$. Function $X(\omega)$ generates the probability measure $P_{X}(B)$ on $B \in \mathrm{B}$ by relation $P_{X}(B)=P\left(X^{-1}(B)\right)$. The function $P_{X}(B)$ is called distribution function of $m$-adic random variable $X . X(\omega)$ is continuous, if there exists integrable on any $B \in \mathrm{B}$ function $f(x)$ such that $P_{X}(B)=\int_{B} f(x) d_{m} x$. We shall call function $f(x)$ density of distribution function of $m$-adic random variable $X$. In the simplest case it is 
possible to choose $\Omega \equiv \mathrm{Q}_{m}$ as the probability space $\Sigma \equiv \mathrm{B}, P(B) \equiv \int_{B} f(x) d_{m} x$ for any $B \in \mathrm{B}$ and some function $f(x)$ such that $f(x) \in L_{\mathrm{loc}}^{1}\left(\mathbf{Q}_{m}\right), f(x) \in L^{1}\left(\mathbf{Q}_{m}\right)$ and $\int_{\mathbf{Q}_{m}} f(x) d_{m} x=1$. In such case $X(\omega)=\omega, \omega \in \mathbf{Q}_{m}$, and random variable $X$ is uniquely defined by the density of distribution function $f(x)$.

Random variable $X$ with the values in $\mathrm{Q}_{m}$ is called infinitely divisible if for any $n \geq 1$ it is possible to find independent identically distributed random variables $X_{n 1}, \ldots, X_{n n}$ with the values in $\mathrm{Q}_{m}$ such that $X=X_{n 1}+\ldots+X_{n n}$.

Characteristic function $\mu(t) m$-adic random variable is defined as

$$
\hat{f}(k)=\int_{\mathbf{Q}_{m}} f(x) \chi(k x) d_{m} x .
$$

Theorem 5.1. Characteristic function of any infinitely divisible m-adic distribution can be represented in the form of (analogous to Levi-Hinchin formula)

$$
\hat{f}(k)=\chi\left(k x_{0}\right) \Omega\left(|k|_{m} p^{-r}\right) \exp \left(\int_{\mathbf{Q}_{m} \backslash\{0\}}(\chi(k x)-1) W(x) d_{m} x\right),
$$

where $x_{0} \in \mathbf{Q}_{m}, r$ is integer, $W(x) d_{m} x$ is the measure on $\mathbf{Q}_{m}$, finite on $\mathbf{Q}_{m} \backslash B_{s}$ for any s, for which $\int_{\mathbf{Q}_{m} \backslash\{0\}}(1-\operatorname{Re\chi }(k x)) W(x) d_{m} x<\infty$ and here $\int_{\mathbf{Q}_{m} \backslash\{0\}} \cdots d_{m} x=$ $\lim _{r \rightarrow-\infty} \int_{\mathbf{Q}_{m} \backslash B_{r}} \cdots d_{m} x$ is the principal value of the integral.

The proof of the Theorem 5.1 coincides with the proof of analogous theorem for totally disconnected locally compact Abel groups (e.g. refer to [19]) also for the field $Q_{p}[20$.

Let us notice that the homogeneous distributions and rotation-invariant temporally and spatially homogeneous processes on $\mathbf{Q}_{p}^{n}$ with independent increments were considered in resent paper 21.

By $m$-adic number random process we shall call mapping $X=X(t, \omega)$ of $\mathbf{R}_{+} \otimes \Omega$ on $\mathbf{Q}_{m}$ which is $\Sigma \mid$ B-measurable for any $t \in \mathbf{R}_{+}$.

By Levi process $X(t)$ from $\mathbf{Q}_{m}$ we shall call stochastically continuous Markov process with stationary independent increments, beginning from zero and having right continuous trajectories with left side limits, i.e. process for which the following conditions are hold:

1. $X(0)=0$ almost everywhere.

2. For any $0 \leq t_{1}<\ldots<t_{n}$ increments $X\left(t_{1}\right), X\left(t_{2}\right)-X\left(t_{1}\right), \ldots, X\left(t_{n}\right)-X\left(t_{n-1}\right)$ are independent.

3. For any $t$ and $s X(t+s)-X(s)=X(t)-X(0)$.

4. For any $t \geq 0$ and $r \lim _{s \rightarrow t} P\left(|X(s)-X(t)|_{m}>p^{r}\right)=0$.

5. Trajectories $X(t)$ for all $t>0$ almost everywhere right continuous and possess left limits. 
From the definition of Levi process it follows that $X(t)$ is infinitely divisible. Characteristic function of Levi process is defined as

$$
\hat{f}(k, t)=\mathrm{M}[\chi(k X(t))]
$$

and due to uniformity and independence of increments has the form

$$
\hat{f}(k, t)=\exp (t \psi(k))
$$

where the form of the function $\psi(k)$ follows from (5.1)

$$
\psi(k)=\int_{\mathbf{Q}_{m} \backslash\{0\}}(\chi(k x)-1) W(x) d_{m} x
$$

It is not difficult to see that $\hat{f}(k, t)=\exp \left(t \int_{\mathbf{Q}_{m} \backslash\{0\}}(\chi(k x)-1) W(x) d_{m} x\right)$ satisfies the equation

$$
\frac{d}{d t} \hat{f}(k, t)=\hat{f}(k, t) \int_{\mathbf{Q}_{m} \backslash\{0\}}(\chi(k x)-1) W(x) d_{m} x .
$$

From (5.2) the equations for the density of distribution function for the Levi process follows

$$
\frac{d}{d t} f(x, t)=\int_{\mathbf{Q}_{m} \backslash\{0\}} W(y-x)(f(y, t)-f(x, t)) d_{m} y
$$

Thus the following theorem takes place.

Theorem 5.2. Distribution function $f(x, t)$ for all Levi process with the values in $\mathbf{Q}_{m}$ satisfies eq. (5.3) where $W(x) d_{m} x$ is the measure on $\mathbf{Q}_{m}$ finite on $\mathbf{Q}_{m} \backslash B_{s}$ for all s, for which $\int_{\mathbf{Q}_{m} \backslash\{0\}}(1-\operatorname{Re\chi }(k x)) W(x) d_{m} x<\infty$.

Note that a particular case of Levi process in $\mathbf{Q}_{m}$ is the one described by Vladimirov equation for which $W(x)=-\frac{1}{\Gamma_{m}(-\alpha)} \frac{1}{|x|^{\alpha}}, \Gamma_{m}(-\alpha)=\frac{1-m^{-\alpha-1}}{1-m^{\alpha}}, \alpha>0$ :

$$
\frac{d f(x, t)}{d t}=-\frac{1}{\Gamma_{m}(-\alpha)} \int_{\mathbf{Q}_{m} \backslash\{0\}} \frac{f(y, t)-f(x, t)}{|y-x|_{m}^{\alpha+1}} d_{m} y .
$$

\section{$6 \quad \mathrm{Q}_{m}$-valued uncoupled continuous time random walk}

Let $\left(\xi_{1}, T_{1}\right),\left(\xi_{1}, T_{1}\right), \ldots$ be a sequence of independent identically distributed pairs of random variables $\xi_{i} \in \mathrm{Q}_{m}$ and $T_{i} \in \mathrm{R}_{+}$. Let the distribution of each pair is given by probability measure $P\left(d_{m} x, d t\right)$ on $\mathbf{Q}_{m} \times \mathrm{R}_{+}$. We shall suppose that $\xi_{i}$ and $T_{i}$ 
are independent and hence $P\left(d_{m} x, d t\right)=\varphi(x) d_{m} x \psi(t) d t$ where $\varphi(x)$ and $\psi(t)$ are probability densities of $\xi_{i}$ and $T_{i}$ respectively. Let us define random process $N(t)$ as

$$
N(t)=\max \left\{n: \sum_{i=1}^{n} T_{i} \leq t\right\}
$$

We shall call the process

$$
X(t)=\sum_{i=1}^{N(t)} \xi_{i}
$$

as $m$-adic uncoupled continuous time random walk ( $m$-adic CTRW).

Let us denote $\sum_{i=1}^{n} T_{i} \equiv t_{n}$. Distribution $p(t, n)$ for $N(t)$ is the measure function $p(t, n)=P(A)$ where $\Sigma \supset A=\left\{\{\omega\}: \forall \omega \in A, t_{n}(\omega)<t \leq t_{n+1}(\omega)\right\}$ or, equivalently as mathematic expectation $p(t, n)=\mathrm{M}\left[I\left(t_{n}<t \leq t_{n+1}\right)\right]$ where

$$
I\left(t_{n}<t \leq t_{n+1}\right)=\left\{\begin{array}{l}
1, t_{n}<t \leq t_{n+1} \\
0, t_{n} \geq t \text { or } t>t_{n+1} .
\end{array}\right.
$$

Let $\hat{p}(s, n)=L[p(t, n)](s)$ be Laplace image of $p(t, n)$. Then

$$
\hat{p}(s, n)=\mathrm{M}\left[\int_{0}^{\infty} d t e^{-s t} I\left(t_{n}<t<t_{n+1}\right)\right]=\mathrm{M}\left[\frac{e^{-s t_{n+1}}-e^{-s t_{n}}}{s}\right] .
$$

Because $t_{n}=\sum_{i=1}^{n} T_{i}$ is the sum of independent random variables, we have

$$
\mathrm{M}\left[e^{-s t_{n}}\right]=\mathrm{M}\left[\exp \left(-s \sum_{i=1}^{n} T_{i}\right)\right]=\hat{\psi}^{n}(s)
$$

where $\hat{\psi}(s)=L[\psi(t)](s)$. Thus

$$
\hat{p}(s, n)=\mathrm{M}\left[\frac{e^{-s t_{n+1}}-e^{-s t_{n}}}{s}\right]=\hat{\psi}^{n}(s) \frac{1-\hat{\psi}(s)}{s}
$$

Distribution density $f(x, t)$ of a process $X(t)$ is Fourier image of characteristic function of the process $X(t)$

$$
f(x, t)=F^{-1}[\tilde{f}(k, t)] .
$$

Using (6.2), we obtain

$$
\tilde{f}(k, t)=\mathrm{M}\left[\chi\left(k \sum_{i=1}^{N(t)} \xi_{i}\right)\right]=\sum_{i=0}^{\infty} \tilde{\varphi}^{n}(k) p(n, t),
$$

where $\tilde{\varphi}(k)=\mathrm{M}\left[\chi\left(\xi_{i} k\right)\right]$ is characteristic function of independent identically distributed variables $\xi_{i}$. Using (6.3) we have

$$
\hat{\tilde{f}}(k, s)=\frac{1-\hat{\psi}(s)}{s} \frac{1}{1-\hat{\psi}(s) \tilde{\varphi}(k)} .
$$


Formula (6.4) is Monthroll-Weiss equation 22 for $m$-adic CTRW with independent jumps. After simple transformations, the equation (6.4) can be represented in an alternative form

$$
\hat{\Phi}(s)[s \hat{\tilde{f}}(k, s)-1]=[\tilde{\varphi}(k)-1] \hat{\tilde{f}}(k, s),
$$

where

$$
\hat{\Phi}(s)=\frac{1-\hat{\psi}(s)}{s \hat{\psi}(s)} .
$$

Inverting Laplace-Fourier transforms, it is possible to rewrite eq. (6.5) in the following forms:

1. Integral equation

$$
f(x, t)=\Psi(t) \delta(x)+\int_{0}^{t} \psi\left(t-t^{\prime}\right) d t \int_{\mathbf{Q}_{m}} \varphi\left(k-k^{\prime}\right) f(x, t) d_{p} k, \Psi(t)=L^{-1}\left[\frac{1-\hat{\psi}(s)}{s}\right] .
$$

2. Generalized Kolmogorov-Feller equation

$$
\int_{0}^{t} \Phi\left(t-t^{\prime}\right) \frac{d}{d t^{\prime}} f(x, t) d t^{\prime}=\int_{\mathbf{Q}_{m}} d_{p} x^{\prime} \varphi\left(x-x^{\prime}\right)\left(f\left(x^{\prime}, t\right)-f(x, t)\right), \Phi(t)=L^{-1}[\hat{\Phi}(s)] .
$$

For exponential distribution of time intervals between the jumps

$$
\psi(t)=e^{-\lambda t}, \psi(s)=\frac{\lambda}{s+\lambda}, \Phi(t)=\frac{1}{\lambda} \delta(t)
$$

the process (6.2) is Levi process with finite measure function $W(x) d_{m} x \equiv \lambda \varphi(x) d_{m} x$ on the whole $\mathbf{Q}_{m}$.

\section{Diffusive limit of $\mathrm{Q}_{m}$-valued CTRW and rela- tionship with Vladimirov equation}

Let us multiply all waiting times $T_{i}$ by the factor $r$, and $\xi_{i} \in \mathbf{Q}_{m}$ - by the factor $h \in \mathbf{Q}_{m}$. Then the distribution densities $\psi(t)$ and $\varphi(x)$ are transformed into

$$
\psi_{r}(t)=r^{-1} \psi\left(\frac{t}{r}\right), \varphi_{h}(x)=|h|_{p}^{-1} \varphi\left(\frac{x}{h}\right) .
$$

For Laplace and Fourier transforms of $\psi(t)$ and $\varphi(x)$ we have

$$
\hat{\psi}_{r}(s)=\hat{\psi}(r s), \tilde{\varphi}_{h}(k)=\tilde{\varphi}(h k) .
$$

Then Monthroll-Weiss equation (6.4) will have the form

$$
\hat{\tilde{f}}_{r, h}(k, s)=\frac{1-\hat{\psi}_{r}(s)}{s} \frac{1}{1-\hat{\psi}_{r}(s) \tilde{\varphi}_{h}(k)}=\frac{1-\hat{\psi}(r s)}{s} \frac{1}{1-\hat{\psi}(r s) \tilde{\varphi}(h k)}
$$


We propose that $\hat{\psi}(s)$ and $\tilde{\varphi}(k)$ have the following asymptotic behavior at $s \rightarrow 0$, $|k|_{m} \rightarrow 0$ :

$$
\hat{\psi}(s)=1-s^{\beta}+o\left(s^{\beta}\right), 0<\beta \leq 1, \tilde{\varphi}(k)=1-|k|_{m}^{\alpha}+o\left(|k|_{m}^{\alpha}\right), \alpha>0
$$

Then we define diffusive limit of $m$-adic CTRW (similarly to definition of diffusive limit of real value CTRW [23, 24]) as

$$
r \rightarrow 0,|h|_{m} \rightarrow 0 \quad \text { on condition that } r^{\beta}=|h|_{m}^{\alpha} .
$$

It is not difficult to see that

$$
\hat{\tilde{f}}_{r, h}(k, s) \rightarrow \hat{\tilde{u}}(k, s) \equiv \frac{1}{s^{\beta}+|k|_{m}^{\alpha}} .
$$

In this passage to the limit, $\hat{\tilde{f}}_{r, h}(k, s)$ and $\hat{\tilde{u}}(k, s)$ are asymptotically equivalent in the Laplace-Fourier domain. Then, the asymptotic equivalence in the $\mathbf{Q}_{m}$-space - real time domain between the $f_{r, h}(x, t)$ and $u(x, t) \equiv F^{-1} L^{-1}[\hat{\tilde{u}}(k, s)]$ is ensured by the continuity theorem for sequences of characteristic functions, after the application of the analogous theorem for sequences of Laplace transforms [25]. Exactly in the same way as in the case of diffusive limit of real valued CTRW, it is possible to show that (ref. [23, 24]) that

$$
f_{r, h}(x, t) \rightarrow u(x, t)
$$

Precise evaluations show that

$$
\begin{gathered}
u(x, t)=F^{-1} L^{-1}[\hat{\tilde{u}}(k, s)]=\int_{\mathbf{Q}_{m}} \chi(-k x) E_{\beta}\left(-|k|_{m}^{\alpha} t^{\beta}\right) d_{m} k= \\
=\left(1-m^{-1}\right)|x|_{m}^{-1} \sum_{i=0}^{\infty} m^{-i} E_{\beta}\left(-m^{-i \alpha}|x|_{m}^{-\alpha} t^{\beta}\right)-|x|_{m}^{-1} E_{\beta}\left(-m^{\alpha}|x|_{m}^{-\alpha} t^{\beta}\right),
\end{gathered}
$$

where

$$
E_{\beta}(z)=\sum_{n=0}^{\infty} \frac{z^{n}}{\Gamma(\beta n+1)}
$$

is Mittag-Leffler function. Function (7.1) satisfies the equation

$$
\frac{\partial^{\beta}}{\partial t^{\beta}} u(x, t)=-\frac{1}{\Gamma_{m}(-\alpha)} \int_{\mathbf{Q}_{m}} \frac{u(y, t)-u(x, t)}{|x-y|_{m}^{\alpha+1}} d_{m} y,
$$

where $\frac{\partial^{\beta}}{\partial t^{\beta}}$ is the fraction Caputo derivative [26]

$$
\frac{\partial^{\beta}}{\partial t^{\beta}} f(t) \equiv \frac{1}{\Gamma(1-\beta)}\left(\frac{\partial}{\partial t} \int_{0}^{t} \frac{f(\tau)}{(t-\tau)^{\beta}} d \tau-t^{-\beta} f(0)\right) .
$$

We shall call equation (17.2) the equation of fractional-time $m$-adic random walk. For $\beta=1$, eq. (7.2) becomes the $m$-adic Vladimirov equation (5.4). 


\section{The equation of fraction-time $m$-adic random walk and asymptotic behavior of the probabil- ity measure of initial distribution support}

We consider the equation of fractional-time $m$-adic random walk (7.2) for $0<\beta \leq$ $1, \alpha>0$ with the initial condition $u(x, 0)=\Omega\left(|x|_{m} \leq 1\right)$. Solution of eq. (17.2) is easy to find using Laplace-Fourier transformations:

$$
\begin{gathered}
u(x, t)=\Omega\left(|x|_{m} \leq 1\right)\left(1-m^{-1}\right) \sum_{i=0}^{\infty} m^{-r} E_{\beta}\left(-m^{-\alpha i} t^{\beta}\right)+ \\
+\Omega\left(|x|_{m}>1\right)\left(\left(1-m^{-1}\right)|x|_{m}^{-1} \sum_{i=0}^{\infty} m^{-i} E_{\beta}\left(-m^{-\alpha i}|x|_{m}^{-\alpha} t^{\beta}\right)-|x|_{m}^{-1} E_{\beta}\left(-m^{\alpha}|x|_{m}^{-\alpha} t^{\beta}\right)\right) .
\end{gathered}
$$

Probability measure of the support of initial distribution is defined as

$$
S(t)=\int_{Z_{m}} u(x, t) d_{m} x=\left(1-m^{-1}\right) \sum_{i=0}^{\infty} m^{-i} E_{\beta}\left(-m^{-\alpha i} t^{\beta}\right) .
$$

Let us find the estimation of (8.1) at $t \rightarrow \infty$. Note that the function $m^{-x}$ decreases with the increase of $x$, while $E_{\beta}\left(-m^{-\alpha x} t^{\beta}\right)$ increases. Consequently on the segment $i \leq x \leq i+1$ we have in equation

$$
m^{-x} E_{\beta}\left(-m^{-i \alpha(x-1)} t^{\beta}\right) \leq m^{-i} E_{\beta}\left(-m^{-i \alpha} t^{\beta}\right) \leq m^{-(x-1)} E_{\beta}\left(-m^{-\alpha x} t^{\beta}\right) .
$$

Integrating 8.2 over $x$ from $i$ to $i+1$ and then summing by $i$ from 0 to $\infty$, we obtain

$$
\frac{1}{\alpha p \ln p} t^{-\frac{\beta}{\alpha}} \Theta_{\beta}(\alpha, t) \leq S(t) \leq \frac{p}{\alpha \ln p} t^{-\frac{\beta}{\alpha}} \Theta_{\beta}(\alpha, t)
$$

where

$$
\Theta_{\beta}(\alpha, t) \equiv \int_{0}^{t^{\beta}} E_{\beta}(-y) y^{\frac{1}{\alpha}-1} d y, \Theta_{\beta}(\alpha) \equiv \int_{0}^{\infty} E_{\beta}(-y) y^{\frac{1}{\alpha}-1} d y .
$$

Because at $t \rightarrow+\infty$ and $\lambda>0$ the following asymptotic formula holds

$$
E_{\beta}\left(-\lambda t^{\beta}\right)=\frac{\sin (\beta \pi)}{\lambda \pi} \frac{\Gamma(\beta)}{t^{\beta}}(1+o(1)),
$$

the integral in $\Theta_{\beta}(\alpha)$ converges at $\alpha>1$ and we have

$$
\frac{1}{\alpha p \ln p} t^{-\frac{\beta}{\alpha}} \Theta_{\beta}(\alpha)(1+o(1)) \leq S(t) \leq \frac{p}{\alpha \ln p} t^{-\frac{\beta}{\alpha}} \Theta_{\beta}(\alpha)(1+o(1)) .
$$

At $\alpha \leq 1$ when $y \rightarrow+\infty$ we have

$$
E_{\beta}(-y) y^{\frac{1}{\alpha}-1}=\frac{\sin (\beta \pi) \Gamma(\beta)}{\pi} y^{\frac{1}{\alpha}-2}(1+o(1)),
$$


therefore

$$
\int_{0}^{t^{\beta}} E_{\beta}(-y) y^{\frac{1}{\alpha}-1} d y=\left\{\begin{array}{l}
\frac{\sin (\beta \pi) \Gamma(\beta)}{\pi} t^{\beta\left(\frac{1}{\alpha}-1\right)}(1+o(1)), \alpha<1, \\
\frac{\sin (\beta \pi) \Gamma(\beta)}{\pi} \ln t(1+o(1)), \alpha=1 .
\end{array}\right.
$$

Finally we obtain the following asymptotic estimate of $S(t)$

$$
\frac{1}{\alpha p \ln p} R(t) \leq S(t) \leq \frac{p}{\alpha \ln p} R(t)
$$

where at $t \rightarrow+\infty$

$$
R(t)=\left\{\begin{array}{l}
t^{-\frac{\beta}{\alpha}} \Theta_{\beta}(\alpha)(1+o(1)), \alpha>1, \\
\frac{\sin (\beta \pi) \Gamma(\beta)}{\pi} t^{-\beta}(1+o(1)), \alpha<1, \\
\frac{\sin (\beta \pi) \Gamma(\beta)}{\pi} \ln t(1+o(1)), \alpha=1 .
\end{array}\right.
$$

\section{Summary}

In this paper we developed a number of theorems from the $p$-adic [2, 15] into $m$ adic analysis of complex valued function. This allowed us to consider some type of $m$-adic-valued stochastic processes. In particular, the distribution function of any $m$-adic-valued Levy process satisfies the pseudodifferential equation of generalized ultrametric random walk. The class of $m$-adic CTRW random processe was considered and its diffusion limit was obtained. It is well known that diffusion limit of real valued CTRW (fractional-time in general) is the diffusion process. However the trajectories of ultrametric processes are not continuous and any of such process is not diffusion in the traditional definition, because the Lindeberg condition is not hold. But we can define $m$-adic process, being the diffusion limit of $m$-adic CTRW, as diffusion indeed. Nevertheless we do not follow this terminology. We call the diffusion limit of $m$-adic CTRW as fractional-time $m$-adic random walk. The distribution function of such process satisfies the Vladimirov equations with fractional time derivative. We also obtained the fractional-time $m$-adic random walk distributive function in explicit form and check the asymptotic behavior of the probability measure of the initial distribution support at large times.

It is interesting to consider the next generalization of the $m$-adic analysis to polyadic. As it is known [27] for any integers sequence $\ldots, a_{-2}, a_{-1}, a_{0}, a_{1}, a_{2}, \ldots$, $\left(a_{i}>1\right)$ there exist the set of polyadic (a-adic) numbers $\mathbf{Q}_{\{a\}}$ that is the set of formal series $x=\cdots+\frac{1}{a_{-3} a_{-2} a_{-1}} x_{-3}+\frac{1}{a_{-2} a_{-1}} x_{-2}+\frac{1}{a_{-1}} x_{-1}+x_{0}+a_{0} x_{1}+a_{1} a_{0} x_{1}+\ldots$, with $x_{i}=0,1, \ldots, a_{i}-1$. The set $\mathbf{Q}_{\{a\}}$ is local compact abelian commutative group and 
has Haar measure and character group, that admits exact realization of measure, Fourier analysis and detailed consideration of polyadic stochastic processes.

\section{Acknowledgements}

A.Z. is grateful to Igor Volovich, Sergey Kozyrev, Vladik Avetisov, Albert Bikulov, Luigi Accardi and Branko Dragovich for useful discussions. Work was partially supported by grants ADTP 3341, 10854 and RFBR 09-01-12161-ofi-m.

\section{References}

[1] W. H. Schikhof, Ultrametric Calculus. An Introduction to $p$-Adic Analysis (Cambridge Univ. Press, Cambridge, 1984).

[2] V. S. Vladimirov, I. V. Volovich and E. I. Zelenov, p-Adic Analysis and Mathematical Physics (World Scient. Publ., Singapore, 1994).

[3] R.Rammal, G.Toulouse, M.A.Virasoro, Ultrametricity for Physicists. Rev. Mod. Phys. 589 (1986) 765-788.

[4] B.Dragovich, A.Yu.Khrennikov, S.V.Kozyrev, I. V. Volovich, On p-adic mathematical physics. $p$-Adic Numbers, Ultrametric Analysis, and Applications Vol. 1, No 1, (2009) 1-17.

[5] A.T.Ogielski, D.L.Stein, Dynamics on ultrametric spaces, Phys. Rev. Lett., V. 55, (1985) 1634-1637.

[6] V.A.Avetisov, A.Kh.Bikulov, S.V.Kozyrev, V.A.Osipov, p-Adic Models of Ultrametric Diffusion Constrained by Hierarchical Energy Landscapes. J. Phys. A: Math. Gen. 35 (2), (2002) 177-189;

[7] V.A.Avetisov, A.Kh.Bikulov, S.V.Kozyrev, Application of p-adic Analysis to Models of Spontaneous Breaking of Replica Symmetry. J. Phys. A: Math. Gen. 32 (50), (1999) 8785-8791.

[8] V.A.Avetisov, A.Kh.Bikulov, V.A.Osipov, p-Adic Description of Characteristic Relaxation in Complex Systems. J. Phys. A: Math. Gen. v.36, N.15., (2003) 4239-4246.

[9] V.A.Avetisov, A.Kh.Bikulov, A.P.Zubarev, First Passage Time Distribution and the Number of Returns for Ultrametric Random Walks. J. Phys. A: Math. Theor. 42, (2009) 085003 - 085020.

[10] R.N.Mantenga, Hierarchical Structure in Financial Market. Eur. Phys. J. B 11 (1999) 193-197.

[11] R. N.Mantenga and H. E. Stanley, An Introduction to Econophysics. Correlations and Complexity in Finance (Cambridge Univ. Press, Cambridge, 2000). 
[12] A.Kh.Bikulov, A.P.Zubarev, L.V.Kaidalova, Hierarchic Dynamic Model of Financial Market near Crash Points and p-adic Mathematical Analysis. Proc. Samara State Tech. Univ., Iss. 42, (2006) 135-141 (in Russian).

[13] S.V.Kozyrev, A.Yu.Khrennikov, Pseudodifferential Operators on Ultrametric Spaces and Ultrametric Wavelets, Izv. RAN. Ser. Mat., 69:5 (2005), p. 133148.

[14] A. Yu. Khrennikov, Non-Archimedean Analysis: Quantum Paradoxes, Dynamical Systems and Biological Models (Kluwer Acad. Publ., Dordreht, 1997).

[15] V. S. Vladimirov, Tables of integrals of complex-valued functions of $p$-adic arguments, arXiv:mathph/ 9911027.

[16] A. N. Kolmogorov and S. V. Fomin, Elements of the Theory of Functions and Functional Analysis, I, II. (Dover Publ.,Mineola, NY, 1999).

[17] G. E. Shilov, Mathematical Analysis 1, 2 (M.I.T., 1974).

[18] A. N. Shiryaev, Probability (Springer, New York, 1996).

[19] K. R. Parthasarathy, Probability Measure on Metric Spaces (Academic Press Inc., London, 1967).

[20] A. N. Kochubei, Pseudo-Differential Equations and Stochastics over NonArchimedean Fields (Marcel Dekker, New York, 2001).

[21] A.N.Kochubei, p-Adic Spherical Coordinates and Their Applications, p-Adic Numbers, Ultrametric Analysis and Applications, Vol. 1, No. 2, (2009), 157166.

[22] E.W.Montroll, G.H.Weiss, J. Math. Phys. 6, (1965) 167-181.

[23] R. Gorenflo and F. Mainardi, Continuous time random walk, Mittag-Leffler waiting time and fractional diffusion: mathematical aspects, Chap. 4., pp. 93127, in Anomalous Transport: Foundations and Applications, Eds. R. Klages, G. Radons and I.M. Sokolov (Wiley-VCH,Weinheim, Germany, 2008).

[24] E.Scalas, R.Gorenflo, F.Mainardi, Uncoupled Continuous-Time Random Walks: Solution and Limiting Behavior of the Master Equation. Phys. Rev. E 69, (2004) 011107-011114.

[25] W. Feller, An Introduction to Probability Theory and its Applications, Vol. II. (Wiley, New York, 1971).

[26] M.Caputo, F.Mainardi, Riv. Nuovo Cimento (Ser. II), 1, (1971)161-198.

[27] E. Hewitt and K. A. Ross, Abstract Harmonic Analysis (Springer-Verlag, 1987). 